\title{
Correlación y concordancia de la autoclinimetría en artritis reumatoide: revisión sistemática de la literatura y metanálisis
}

\author{
Patients-reported outcomes correlation and concordance in rheumatoid arthritis: \\ systematic literature review and metaanalysis
}

Jenny Amaya-Amaya ${ }^{1}$, Gloria Torralvo-Morato ${ }^{1}$, Omar-Javier Calixto ${ }^{2}$, Rolando Calderón-Rojas², Julián Caro-Moreno², Aura-María Domínguez ${ }^{3}$, Rubén-Darío Mantilla1,3, Juan-Manuel Anaya ${ }^{1}$, Adriana Rojas-Villarraga ${ }^{1}$

Palabras clave:

Autoclinimetría; clinimetría; artritis reumatoide.

Este artículo contiene material complementario al cual se puede acceder a través del link: http://www.rcreumatologia.org/ index.php/rcr/rt/suppFiles/365/0

Recibido:

21 de agosto de 2011

Aceptado:

20 de febrero de 2013

\section{RESUMEN}

Introducción: Existe un interés mundial en la evaluación de la artritis reumatoide (AR) desde la perspectiva del paciente mediante la autoclinimetría [en inglés patient-reported out comes (PROs) ], que busca evaluar la actividad de la enfermedad, calidad de vida, pronóstico y adherencia terapéutica.

Objetivo: Evaluar y analizar de manera objetiva el grado de correlación o concordancia entre las herramientas de autoclinimetría implementadas en AR con las mediciones objetivas realizadas por el equipo de salud, a través de una revisión sistemática de la literatura mundial y metanálisis. Materiales y métodos: Se realizó revisión sistemática de la literatura acerca de autoclinimetría en AR en PubMed, LILACS, Embase, SciELO y Biblioteca Virtual de la Salud, utilizando términos $\mathrm{MeSH}$ y DeCS, con un solo limite utilizado: humanos. Posteriormente, se realizó el metanálisis utilizando el programa Comprehensive Meta-Analysis versión 2.

Resultados: Se incluyeron 85 artículos, demostrando correlaciones de moderadas a altas, entre las medidas objetivas realizadas por profesionales de la salud [DAS28, CDAI, SDAI, conteo articular inflamatorio (CAI) y doloroso (CAD), entre otros],y de autoclinimetría (RAPIDs, RADAI, RADAR, HAQ y CAl y CAD), por medio de metanálisis utilizando modelo de efectos aleatorios $(p<0.0001)$.

Conclusiones: La autoclinimetría tiene correlación y concordancia adecuadas con las medidas objetivas realizadas por el médico y su equipo entrenado. Ésta puede ser administrada tanto en la práctica clínica cotidiana como en ensayos clínicos, sin pretender que remplace el juicio clínico, pero con el ánimo de facilitar y optimizar el tiempo de consulta y los desenlaces de los pacientes.
Key words:

Patient-reported outcomes; clinical assessment; rheumatoid arthritis.
Los autores declaran no presentar ningún conflicto de interés al momento de la redacción del manuscrito.

\section{SUMMARY}

Introduction: There is a worldwide interest about the evaluation of rheumatoid arthritis (RA) from the perspective of the patient, using patient-reported outcomes (PROs), in order to assess disease activity, quality of life, prognosis, and treatment adherence.

Objective: To evaluate and analyze the instruments of PROs and the degree of correlation with the objective measures done by the health practitioners through a systematic review and metaanalysis.

1. Centro de Estudio de Enfermedades Autoinmunes (CREA). Escuela de Medicina y Ciencias de la Salud, Universidad del Rosario, Bogotá, Colombia.

2. Semillero de Investigación CREA. Escuela de Medicina y Ciencias de la Salud. Universidad del Rosario, Bogotá, Colombia.

3. Riesgo de Fractura, CAYRE IPS, Escuela de Medicina y Ciencias de la Salud. Universidad del Rosario, Bogotá, Colombia.

Correspondencia:

Adriana Rojas Villarraga: adrirojas@gmail.com 


\begin{abstract}
Materials and methods: A systematic review was done in PubMed, LILACS, Embase, SciELO y Virtual Library Health (Biblioteca Virtual de la Salud) databases, using the MeSH terms and DeCS, with limit: Humans. A metaanalysis was done using the software Comprehensive MetaAnalysis version 2.

Results: 85 articles were included, finding high to moderate correlations between the objective measures performed by trained health care practitioners [DAS28, CDAI, SDAI, swollen joint count (SJC) and tender joint count (TJC) among others] and PROs (RAPIDs, RADAI, RADAR, HAQ, SJC and TJC) through metaanalysis applying the random effect models $(p<0.0001)$.

Conclusions: PROs have an adequate correlation and concordance with the objective measures done by the health care practitioners. It can be administrated in the clinical practice and clinical trials, without pretending to replace the clinical concept but with the aim to facilitate and optimize the clinical consult and the patient outcomes.
\end{abstract}

\section{Introducción}

La artritis reumatoide (AR) es una patología autoinmune, inflamatoria y crónica, con compromiso autoinmune sistémico, permite la aparición de manifestaciones extra-articulares como. por ejemplo, nodulosis y enfermedad cardiovascular ${ }^{1-3}$. La AR conlleva acomorbilidades ${ }^{4,5}$, discapacidad ${ }^{6,7}$, deterioro en la calidad de vida ${ }^{8,9}$, y mortalidad prematura ${ }^{10,11}$, siendo ésta última dos veces mayor que la de la población general ${ }^{1,12}$.

La frecuencia de presentación varía entre 0,5 y $1 \%$ en las poblaciones estudiadas ${ }^{13,14}$; para los países en vía de desarrollo se ha reportado una prevalencia menor (entre $0.1-0.5 \%$ ) incluyendo población latinoamericana ${ }^{12,13,15}$. En general, en las mujeres se presenta con una frecuencia tres veces superior que la de los hombres $3,5,13,16,17$.

Teniendo en cuenta que la AR es una de las artropatías más frecuentes y que una mala evaluación puede llevar a un tratamiento inadecuado, con desenlaces no deseados, es necesario medir clínicamente y de manera objetiva la actividad de la enfermedad y el grado de afectación de los pacientes. Dicha medición, en la mayoría de los casos, está limitada a la evaluación por el reumatólogo experto, con muchas restricciones, entre las que se destacan la poca disponibilidad de tiempo, la ausencia de un estándar de oro para las mediciones ${ }^{18-20}$ y la falta de participación del paciente ${ }^{21-23}$. En general, la evaluación del paciente se ha centralizado en el médico o en el equipo de salud que lo atiende.

Existe un creciente interés mundial en la evaluación de varias enfermedades desde la perspectiva del paciente, reflejado a su vez en la implementación en la AR mediante la autoclinimetría [en inglés referido como patient-reported outcomes (PROs)], definido como el proceso mediante el cual, a través de la aplica- ción de herramientas e instrumentos de medición por parte del paciente, se realiza la evaluación objetiva de la enfermedad, permitiendo acumular la información necesaria para mejorar la orientación cuantitativa delas decisiones clínicas y terapéuticas, la documentación de la situación y mejorías, así como valorar el desenlace y el pronóstico del paciente ${ }^{18,19,24-30}$.

Cientos de cuestionarios y escalas de autodiligenciamiento para evaluar estas variables, por parte de los pacientes, han sido construidos en las últimas décadas para el uso en la práctica clínica e investigación ${ }^{31,32}$. Todas ellas han sido validadas mundialmente, son confiables, reproducibles ${ }^{27,33-36}$, y parecen ser igual o incluso más informativas que el solo conteo articular $(\mathrm{CA})^{19}$, ya que proveen información valiosa, útil, objetiva y única del estado funcional y clínico informado por el propio paciente ${ }^{37}$.

Es por esto que desde los años 80, la evaluación de los pacientes con AR ha experimentado un cambio, se han involucrado tanto los procesos fisiopatológicos de la enfermedad [ej. medición de proteína $\mathrm{C}$ reactiva (PCR), velocidad de eritrosedimentación (VSG), anticuerpos, radiografías], como la actividad de la enfermedad, capacidad funcional, daño estructural, dolor, fatiga y calidad de vida. Todos ellos permiten una evaluación más objetiva y a largo plazo de los desenlaces más relevantes en cada paciente ${ }^{38}$, como, por ejemplo, comorbilidades, toxicidad medicamentosa, consecuencias sociales y psicológicas, pronóstico, mortalidad prematura y altos costos secundarios ${ }^{2,6,18,21,27,30,33,39,40}$.

Algunos estudios han demostrado correlación entre las medidas autoadministradas y las evaluadas por el médico, entre las que se encuentran el conteo articular doloroso (CAD) e inflamado (CAI), entre otras $^{19,31,41-44}$. Una publicación reciente desarrollada con pacientes colombianos, demostró hallazgos que 
concuerdan con los de la literatura mundial, encontrando correlaciones moderadas a altas entre el índice de actividad de la enfermedad [del inglés disease activityscore $\left(\mathrm{DAS}^{28}\right)$ ] y el CAI y CAD reportado por el paciente, al igual que con la escala visual análoga global del paciente (EVA-G), entre otras ${ }^{45}$. Por el contrario, algunos autores han demostrado una baja correlación entre las mediciones realizadas por el paciente y aquellas realizadas por el médico ${ }^{46,47}$. Aunque algunos autores ${ }^{48}$ han resaltado que es indispensable y prioritario el desarrollo de metanálisis que evalúen los desenlaces en AR (por médico y paciente), son pocos los estudios de este tipo. Barton y cols. ${ }^{49}$ realizaron un metanálisis que evaluó exclusivamente la correlación entre los conteos articulares de médico y paciente, siendo baja para el CAI y moderada para el $\mathrm{CAD}$; en dicho análisis no se incluyeron otros tipos de mediciones (Ej. DAS28, etc). Kalyouncu y cols. ${ }^{50}$ realizaron una revisión sistemática de la literatura que se limita a una descripción con proporciones del tipo de estudios y de escala de autoclinimetría utilizadas, sin realizar un análisis objetivo de la relación entre estas. Por todo lo anterior, se considera necesario e imperativo un metanálisis que agrupe las diferentes medidas de clinimetría (médico y paciente), y que evalúe de manera objetiva la correlación y concordancia.

En el presente estudio se evaluará y analizará el grado de correlación o concordancia entre las herramientas de autoclinimetría (PROs) implementadas en AR con las mediciones objetivas realizadas por el médico (o el equipo de salud) a través de una revisión sistemática de la literatura mundial y metanálisis.

\section{Materiales y métodos}

1. Estrategia de búsqueda: Se realizó la revisión sistemática de la literatura acorde con el objetivo del estudio en las siguientes bases de datos: PubMed, LILACS, Embase, SciELO y Biblioteca Virtual de la Salud (BVS). La búsqueda incluyó artículos publicados entre enero de 1955 hasta julio de 2012. Dos investigadores (JAA- OJC) realizaron la búsqueda de manera independiente. Los resultados fueron comparados y los desacuerdos fueron convenidos bajo consenso de los investigadores. La extracción, análisis y reporte de datos se realizaron siguiendo las guías PRISMA (del inglés Preferred Reporting Items for Systematic Reviews and Meta-analyses) ${ }^{51}$.
No hubo límites referentes al idioma, periodo o tipo de publicación. Solo el límite "humano" fue incluido. La búsqueda realizada en PubMed, Scopus y Embase se llevó a cabo teniendo en cuenta el término MeSH (del inglés Medical Subject Headings) "Rheumatoid Arthritis", cruzado con cada uno de los siguientes términos MeSH "Diagnostic Self-Evaluation", "Selfassessment", "Self-examination" y "Self-report", al igual que con las siguientes palabras clave: "Patient-Reported Outcomes", "Self-administered", "Self-assess", "Selfevaluation", "Self-examine", "Self-monitor", "Self-monitoring", "Self-perception", "Self-rate" y "Self-rating".

La misma estrategia anteriormente mencionada se utilizó para la búsqueda en las bases SciELO y BVS. Cada término MeSH fue traducido como término DeCs (descriptores en ciencias de la salud), al español y portugués. Una vez más, no hubo límites en idioma y tipo de publicación. Los términos empleados fueron: "Artrite Reumatóide" y "Artritis Reumatoidea", con cada uno de los siguientes: "Autoexamen", "Auto-Exame", "Autoinforme", "Auto-Relato", "Autorreporte", "Autoevaluación diagnóstica", "Auto-Avaliação Diagnóstica”, "Autoevaluación", "Auto-Avaliação", "Autodiagnóstico", "Auto-diagnosis" y "Diagnóstico mediante Autoevaluación". También se utilizaron como palabras claves "Autoclinimetría" y "Clinimetría". Adicionalmente, se realizó una búsqueda en las bases de datos correspondientes a los principales congresos del tema en el mundo [American College of Rheumatology (ACR) y European League Against Rheumatism (EULAR)].

La búsqueda no se limitó por términos relacionados con el diseño del estudio (Ej. "correlation" "concordance", etc.), para obtener así el mayor número de artículos en la fase de identificación y luego proceder en la fase de tamizaje a seleccionar aquellos con las variables y diseño de interés. De los artículos seleccionados, se revisaron, manualmente, una a una las referencias, para obtener el $100 \%$ de los artículos.

2. Selección de estudios, extracción de datos y evaluación de calidad: Los criterios fueron aplicados de manera exacta por los dos revisores. Un estudio se incluía si: a) el resumen se encontraban disponible, b) contenía datos originales, c) utilizaba una clasificación aceptada de criterios para AR, d) reportaba índices de correlación o concordancia entre las mediciones objetivas del médico y las reportadas por el paciente. Los artículos excluidos del análisis fueron: los que 
evaluaban artritis idiopática juvenil, modelos animales, reportes de casos, editoriales, cartas al editor, no evaluaban los datos de interés, realizaban mediciones de índole psicológica o no realizaban análisis estadístico. En primera instancia se revisaron títulos y resúmenes, en busca de los criterios de elegibilidad. De los que eran elegibles se revisó el texto completo. Aquellos artículos que parecían ser relevantes para el presente metanálisis, provenientes de las referencias de artículos seleccionados y que no se encontraron en la búsqueda inicial también se obtuvieron (búsqueda manual). La estrategia de búsqueda contempló contactar a los autores vía correo (electrónico o postal), si el texto del artículo seleccionado no se encontraba completo, luego de hacer una exploración exhaustiva en las diferentes bases de datos. Si los artículos seleccionados se encontraron en otro idioma, diferente al español, portugués o inglés, se realizó la traducción oficial del resumen y, de acuerdo con esto, se decidió la inclusión del artículo completo. Durante el proceso, los artículos duplicados fueron excluidos. Si el tipo de estudio correspondía a revisión tanto sistemática como no sistemática y metanálisis, se realizó una exploración detallada de todas las referencias incluidas para verificar la inclusión de los artículos allí mencionados ${ }^{38,49,50,52-54}$. No se contempló la inclusión de los datos de estos análisis para evitar duplicidad de valores, ya que se consideró incluir los datos de los estudios originales para efectos de metaanalizarlos.

Cuatro revisores, de manera independiente (GTM, OJC, RCR, JCM), organizaron los artículos seleccionados, basados en el país de origen y ańo de publicación, tipo de publicación, tamaño de muestra, tipo de mediciones objetivas y subjetivas obtenidas (médico vs. autoclinimetría), tipo de correlación o concordancia y su respectiva significancia estadística. Posterior a ello, cuando los revisores encontraban desacuerdos, un quinto revisor (JAA) se encargó de llegar a consenso con cada uno de ellos. Se realizó un análisis descriptivo y detallado de los datos encontrados. Los artículos no fueron incluidos si no cumplían los criterios de inclusión o si no tenían los datos pertinentes.

Se construyó una base de datos con la información pertinente, que incluye autor, país, año, lenguaje, diseño del estudio, número de pacientes. El desenlace que se extrajo fue el tipo y nivel de correlación o concordancia según el caso, valor p y las medidas entre las cuales se realizó la correlación o concordancia, clasificados de acuerdo con la medición que se pretendía realizar en cada estudio (Ej. calidad de vida, actividad de la enfermedad, etc.). La calificación de calidad de las publicaciones (material suplementario 1) fue realizada mediante los niveles establecidos por el "Oxford Centre for Evidence-Based Medicine" de $2011^{55}$. Ningún artículo fue excluido de la revisión por esta calificación.

3. Metanálisis: Los datos fueron analizados utilizando el programa Comprehensive Meta-Analysis versión 2 (Biostat, Englewood, NJ, 2004). Los cálculos se realizaron teniendo en cuenta el grupo total de artículos dependiendo de los datos disponibles. No se tuvieron en cuenta para el análisis, el tipo de intervenciones o tratamientos, dado que se incluyó el nivel de correlación o concordancia informado por cada publicación entre las diferentes escalas o herramientas (autoclinimetría y médico), de manera independiente a la intervención, teniendo en cuenta que ambas mediciones se realizan bajo las mismas condiciones (Ej. Con o sin tratamiento).

El tamaño del efecto se calculó de dos maneras: en el primer abordaje se incluyeron todos los estudios que brindaron el coeficiente de correlación con el respectivo tamaño de la muestra. En aquellos estudios en que no se disponía del coeficiente, se tuvo en cuenta el valor p y el tamaño de la muestra. En ambos escenarios se incluyó el dato de la dirección de la correlación (Ej. positiva, negativa, no indicada). Los estudios se clasificaron de acuerdo con tres parámetros: el estadístico utilizado para el cálculo de la correlación (Ej. Pearson, Spearman, etc.), la medición de clinimetría realizada por el observador objetivo (médico o equipo de la salud) y la medición de autoclinimetría desarrollada por el paciente. Adicionalmente, se tuvieron en cuenta aquellos estudios que hubieran realizado el cálculo de asociación entre las dos variables a través de una regresión lineal múltiple, con su respectivo tamaño de la muestra.

En el segundo abordaje, de manera independiente, el tamaño del efecto fue calculado con aquellos estudios que aportaron el cálculo de concordancia, de igual modo teniendo en cuenta el tamaño de la muestra.

En ambos casos, los resultados se graficaron por medio del diagrama de bosque (forest plot). En este metanálisis se tuvieron en cuenta estudios con diseños 
diferentes para calcular el mismo tamaño del efecto, teniendo en cuenta que el tamańo del efecto tiene el mismo significado en todos los estudios así como puede ser comparable, a través de ellos, de manera relevante. Se ha determinado que para los estudios que informan correlaciones entre dos variables, la correlación de por sí, sirve como el tamaño del efecto índice y ya ha sido estandarizada para tener en cuenta diferentes métricas en las escalas originales; todos los valores de correlación fueron convertidos a una escala $\mathrm{Z}$ de Fisher y todos los análisis se realizaron con los valores transformados; el resultado final y su intervalo de confianza fueron convertidos de nuevo para presentar los datos en valores de correlaciones.

Para cada metanálisis se realizó un análisis de sensibilidad para comparar los resultados, incluyendo y excluyendo los diferentes estudios (uno a la vez), determinando así la solidez de las conclusiones. Adicionalmente, se realizó un análisis acumulativo, para evidenciar el peso de cada estudio dentro del análisis global.

Se realizaron metanálisis adicionales, teniendo en cuenta estudios específicos que tuvieran datos de estructura compleja, con resultados diferentes no acumulativos, y en donde la información para calcular los diferentes tamańos del efecto fuera del todo independiente, esto es, estudios que realizaron correlaciones entre diferentes medidas de observador y paciente, respectivamente, pero independientes entre sí.

Para cada análisis, el efecto final medido a través del índice de correlación y su intervalo de confianza (IC) 95\%, fue obtenido por el modelo de efectos aleatorios, porque todos los estudios fueron considerados desiguales en términos de mediciones realizadas, más aun, dado que este modelo asume que existe una distribución del verdadero tamaño del efecto. Se realizaron análisis incluyendo todos los estudios que evaluaran la misma medida de clinimetría tanto por el observador como por el paciente (Ej. CAI observador vs. CAI paciente). Adicionalmente, se compararon todas aquellas medidas realizadas por el observador, correlacionadas con una medición específica en el paciente y viceversa (Ej. DAS28 por el observador vs. todas las mediciones de autoclinimetría correlacionadas y publicadas con éste). Se realizó análisis por comparación de grupos, teniendo en cuenta el estadístico utilizado para el cálculo de la correlación, de modo que se compararon los tamańos del efecto de acuerdo con cada grupo de artículos que utilizaron un mismo estadístico (Ej. Pearson vs. Kendall). Los mismos procedimientos se realizaron para aquellos análisis que tuvieron en cuenta la concordancia. Adicionalmente, se realizaron análisis de acuerdo con el número de visitas (estudios longitudinales), teniendo en cuenta cada visita y punto de corte, de manera independiente, si el estudio correspondía a pacientes bajo intervenciones con medición en diferentes momentos del seguimiento.

La heterogeneidad, tanto para los metanálisis de correlaciones y aquellos de concordancias, se calculó por medio de las pruebas Higgins $\left(\mathrm{I}^{2}\right)$ y Q de Cochran (Q).

La prueba $\mathrm{I}^{2}$ muestra la proporción de la dispersión real observada más que la dispersión espuria y se expresó en porcentaje con un rango de $0 \%$ a $100 \%$. Los valores de $\mathrm{I}^{2}$ fueron cualitativamente calificados como bajo (25\%), medio (50\%) y alto $(75 \%)$. Se consideró un valor del estadístico $\mathrm{Q}$ significativo $(\mathrm{p}<0.10)$ como indicativo de heterogeneidad entre los estudios. Se calculó la heterogeneidad entre los estudios y entre los grupos de estudios, cuando se agruparon por medio del estadístico utilizado. El sesgo de publicación se determinó utilizando el gráfico de embudo (funnel plot) y el test de asimetría de regresión de Egger. Se realizaron pruebas adicionales si estos dos indicaban sesgo de publicación. Por último se realizó un análisis acumulativo para evaluar el peso de los diferentes estudios.

\section{Resultados}

1. Revisión sistemática de la literatura. Se identificaron 5.455 artículos de PubMed, 8.442 de Scopus, y 9.469 de Embase. De las bases de datos latinoamericanas, se obtuvieron 4.514 de BVS y 4 de SciELO, para un total de 27.884 potenciales artículos. De estos, 22.848 fueron encontrados como duplicados, quedando como artículos elegibles un total de 5.036. Según la revisión de título y resumen se obtuvieron 418 artículos potenciales, de los cuales 414 fueron revisados en texto completo. Por búsqueda manual, extrayendo referencias de los artículos seleccionados y a través de los resúmenes de los dos congresos mencionados se obtuvieron 10 artículos más. No se requirió traducción oficial de artículo alguno. 
Finalmente un total de 85 fueron tenidos en cuen-

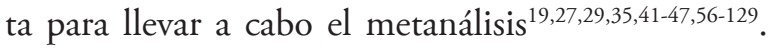
El resumen de 4 artículos fue revisado, pero no se pudieron extraer datos, dado que no fue posible su consecución en texto completo aun después de escribir a sus respectivos autores ${ }^{130-133}$.

La distribución geográfica fue: 1 de Argentina, 9 de Austria, 1 de Canadá, 2 de Chile, 1 de Colombia, 2 de Egipto, 1 de España, 31 de Estados Unidos de América, 1 de Estonia, 1 de Finlandia, 4 de Francia, 9 de Holanda, 1 de Italia, 2 de Marruecos, 2 de Noruega, 1 de Nueva Zelanda, 1 de Polonia, 6 del Reino Unido, 1 de Singapur, 3 de Suecia, 3 de Suiza y 2 de Turquía. La distribución de acuerdo con los tipos de estudio fue: 53 de corte transversal, 30 cohortes y 2 ensayos clínicos. Aproximadamente $62 \%$ tienen un tamaño de muestra mayor a 100 pacientes. El flujograma de la revisión sistemática y los artículos incluidos en el análisis se muestra en la Figura 1.

Dentro de las medidas objetivas, a pesar de ser 85 artículos seleccionados, para el CAI se encontraron 156 mediciones, para el CAD 181 y para el CA sin discriminación fueron halladas 30 mediciones. El DAS fue valorado en 124 oportunidades, el índice clínico de actividad de la enfermedad [del inglés Clinical Disease Activity Index (CDAI)] fue valorado en 45 oportunidades, mientras que el índice sim- plificado de actividad de la enfermedad [del inglés Simplified Disease Activity Index (SDAI)] fue medido en 39 oportunidades. El cuestionario de evaluación de salud [del inglés Health Assessment Questionnaire (HAQ)] realizado por el médico o personal de la salud entrenado, fue aplicado en 17 oportunidades. Otros que fueron tenidos en cuenta como medición objetiva fueron el índice articular de Thomson (Thom), el índice articular de Richie (RAI), la EVA-G, entre otros, el total fue de 71 mediciones. En cuanto a las medidas realizadas por el paciente, se encontraron las siguientes valoraciones: CA en 15 oportunidades, CAD en 120 , CAI 141, DAS en 23 ocasiones, HAQ y MDHAQ (del inglés Multidimensional Health Assessment Questionnaire) 108 veces, el índice de actividad de la enfermedad en artritis reumatoide [del inglés Rheumatoid Arthritis Disease Activity Index (RADAI)]) 46 veces, datos de índice de evaluación rutinaria de los pacientes RAPID 3, RAPID 4 y RAPID5 [del inglés (Routine Assessment of Patients Index Data-RAPID)] en 46, 10 y 10 mediciones, respectivamente. La evaluación rápida de la actividad de la enfermedad en reumatología, [del inglés Rapid Assessment Disease Activityin Rheumatology (RADAR)], fue realizada 26 veces. La escala visual análoga del dolor del paciente (EVA-D) fue medida 39 veces y la EVA-G en 45 oportunidades. Por su parte, el cálculo del SDAI y CDAI, a partir del

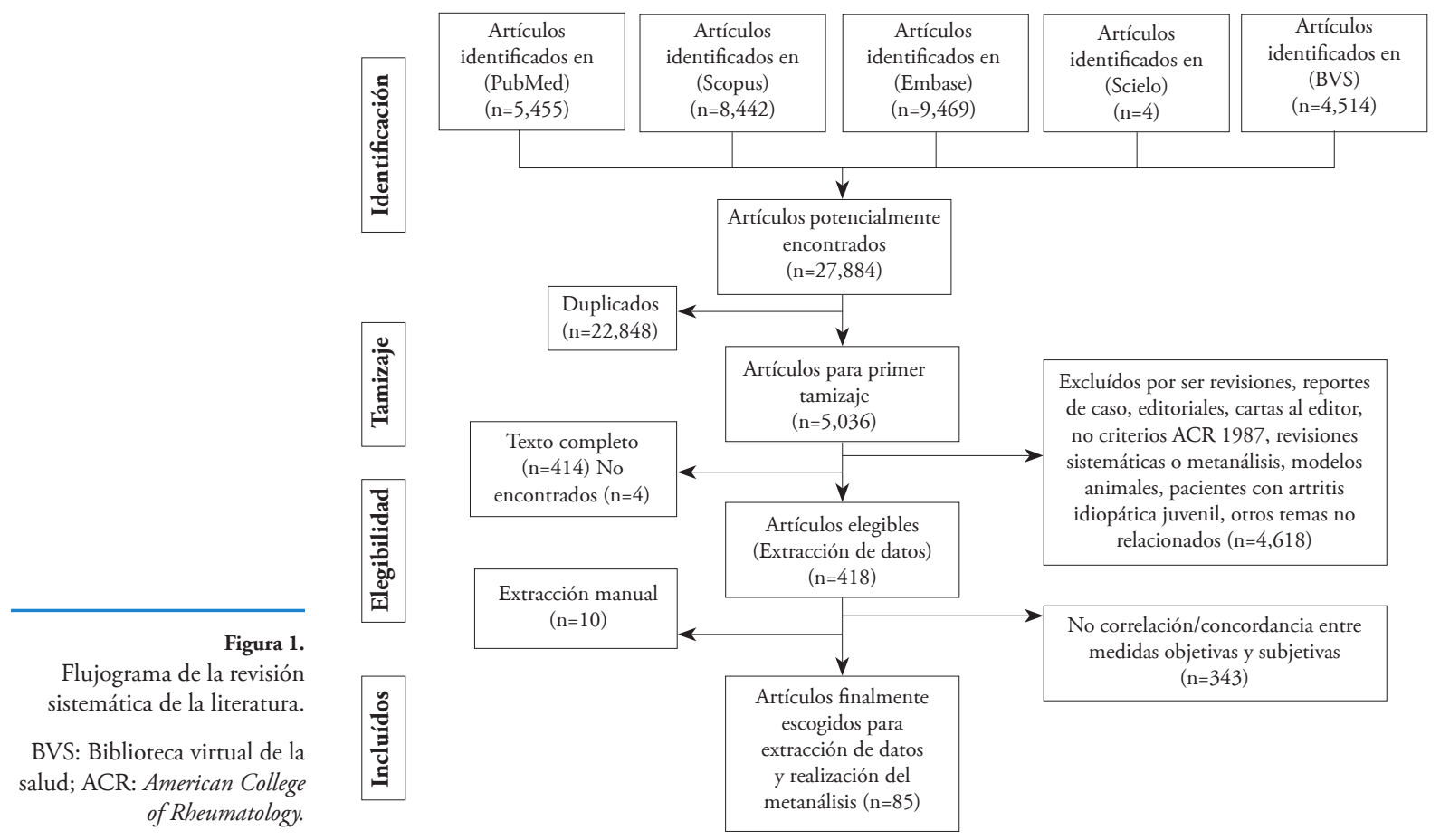


conteo articular del paciente,se realizaron en 3 ocasiones cada uno. Otros que fueron considerados como medidas subjetivas fueron el RAI, Thom, realizados por el paciente, índices de funcionalidad, otras escalas de dolor, índices de realización de actividades diarias (AVD), calidad de vida e índices de percepción de la enfermedad, fueron valorados en 27 oportunidades. Los valores de todas las correlaciones y concordancias se encuentran en el material suplementario 1.

2. Metanálisis: Para el primer tamaño final del efecto basado en correlaciones se desarrollaron 33 metanálisis (incluyendo aquellos que compararon subgrupos de acuerdo con el estadístico), de los cuales todos mostraron una correlación directa entre las medidas objetivas realizadas por el observador y aquellas de autoclinimetría desarrolladas por el paciente, con diferentes grados de correlación. En la tabla 1 se encuentran los resultados del tamaño final del efecto basados en el modelo de efectos aleatorios, con el respectivo IC 95\%, el número de estudios y subgrupos tenidos en cuenta en cada análisis, así como los datos relevantes de heterogeneidad y prueba de sesgo de publicación. Las figuras 2 y 3 muestran los diagramas de bosque de la correlación entre CAI (observador vs. paciente) y CAD (observador vs. paciente). En la figura 4 se muestra el diagrama de bosque evaluando la correlación entre el DAS28 evaluado por el observador y por el paciente. En el material suplementario $2 \mathrm{~A}$, se encuentran todos los diagramas de bosque de los metanálisis adicionales, así como los diagramas de análisis de sensibilidad y grá-

\begin{tabular}{|c|c|c|c|c|c|c|c|}
\hline $\begin{array}{l}\text { Medición } \\
\text { objetiva }^{a}\end{array}$ & $\begin{array}{l}\text { Medición } \\
\text { autoclinimetría }\end{array}$ & $\begin{array}{l}\text { Número } \\
\text { de estudios } \\
\text { (subgrupos) }\end{array}$ & $\begin{array}{l}\text { Tamańo } \\
\text { del efecto } \\
\text { final b }\end{array}$ & IC 95\% & Valor p & $l^{2}$ & $\begin{array}{l}\text { Valor p } \\
\text { Egger }\end{array}$ \\
\hline $\mathrm{CAI}^{\mathrm{C}}$ & $\mathrm{CAI}^{\mathrm{c}}$ & $15(37)$ & 0.577 & $0.506-0.604$ & $<0.0001$ & 80 & 0.31 \\
\hline $\mathrm{CAI}^{\mathrm{d}}$ & $\mathrm{CAI}^{\mathrm{d}}$ & $15(31)$ & 0.555 & $0.492-0.613$ & $<0.0001$ & 82 & 0.23 \\
\hline $\mathrm{CAI}^{\mathrm{e}}$ & $\mathrm{CAI}^{\mathrm{e}}$ & $15(24)$ & 0.543 & $0,479-0,601$ & $<0.0001$ & $81 \mid$ & 0.37 \\
\hline $\mathrm{CAI}^{\mathrm{f}}$ & $\mathrm{CAI}^{\mathrm{f}}$ & $13(24)$ & 0.553 & $0.482-0.617$ & $<0.0001$ & 84 & 0.29 \\
\hline CAI ${ }^{\mathrm{g}}$ & CAI ${ }^{\mathrm{g}}$ & $13(24)$ & 0.521 & $0.452-0.583$ & $<0.0001$ & 82 & 0.780 \\
\hline $\mathrm{CAD}^{\mathrm{c}}$ & $\mathrm{CAD}^{\mathrm{c}}$ & $15(38)$ & 0.715 & $0.663-0.761$ & $<0.0001$ & 91 & $0.017^{\mathrm{h}}$ \\
\hline $\mathrm{CAD}^{\mathrm{d}}$ & $\mathrm{CAD}^{\mathrm{d}}$ & $14(33)$ & 0.735 & $0.676-0.785$ & $<0.0001$ & 91 & 0.06 \\
\hline $\mathrm{CAD}^{\mathrm{e}}$ & $\mathrm{CAD}^{\mathrm{e}}$ & $15(36)$ & 0.629 & $0.556-0.692$ & $<0.0001$ & 92 & 0.57 \\
\hline $\mathrm{CAD}^{\mathrm{f}}$ & $\mathrm{CAD}^{\mathrm{f}}$ & $13(25)$ & 0.682 & $0.611-0.742$ & $<0.0001$ & 91 & 0.49 \\
\hline $\mathrm{CAD}^{\mathrm{g}}$ & $\mathrm{CAD}^{\mathrm{g}}$ & $12(23)$ & 0.706 & $0.635-0.76$ & $<0.0001$ & 92 & 0.24 \\
\hline DAS28 & DAS28 & $5(8)$ & 0.845 & $0.67-0.931$ & $<0.0001$ & 99 & 0.06 \\
\hline DAS28 & Múltiple $^{\mathrm{i}}$ & $30(93)$ & 0.534 & $0.489-0.577$ & $<0.0001$ & 98 & $0.0001^{\mathrm{h}}$ \\
\hline DAS28 & RAPID $(3,4,5)$ & $9(17)$ & 0.625 & $0.590-0.658$ & $<0.0001$ & 70 & 0.088 \\
\hline CDAI & Múltiple & $12(34)$ & 0.656 & $0.573-0.726$ & $<0.0001$ & 97 & $0.0018^{h}$ \\
\hline SDAI & Múltiple $^{\mathrm{i}}$ & $9(39)$ & 0.616 & $0.575-0.653$ & $<0.0001$ & 92 & $0.033^{h}$ \\
\hline Múltiple ${ }^{j}$ & RAPID3 & $8(32)$ & 0.602 & $0.551-0.649$ & $<0.0001$ & 91 & 0.44 \\
\hline Múltiple ${ }^{j}$ & RAPID4 & $3(8)$ & 0.626 & $0.526-0.709$ & $<0.0001$ & 87 & 0.24 \\
\hline Múltiple ${ }^{j}$ & RAPID5 & $2(8)$ & 0.719 & $0.627-0.791$ & $<0.0001$ & 89 & 0.62 \\
\hline Múltiple ${ }^{j}$ & RADAI & $10(43)$ & 0.568 & $0.533-0.60$ & $<0.0001$ & 88 & $0.008^{h}$ \\
\hline Múltiple ${ }^{j}$ & RADAR & $4(22)$ & 0.470 & $0.402-0.533$ & $<0.0001$ & 55 & 0.65 \\
\hline Múltiple ${ }^{j}$ & HAQ & $24(75)$ & 0.481 & $0.431-0.527$ & $<0.0001$ & 97 & $<0.0001^{\mathrm{l}}$ \\
\hline Múltiple ${ }^{j}$ & EVA dolor & $17(39)$ & 0.471 & $0.411-0.526$ & $<0.0001$ & 90 & 0.69 \\
\hline Múltiple $^{j}$ & EVA global & $13(40)$ & 0.549 & $0.49-0.602$ & $<0.0001$ & 92 & 0.52 \\
\hline
\end{tabular}

Resultados de los metanálisis con correlación como tamaño final del efecto basados en modelos de efectos aleatorios. a: Medición realizada por observador objetivo del equipo médico; b: correlación basado en modelo de efectos aleatorios; c: incluyendo la medición genérica CA; d: sin incluir la medición genérica CA; e: visita 1; f: visita 2; g: visita 3; h:pruebas complementarias (Trim and fill, Begg y Mazumdar, classic fail-safe) detalladas en material suplementario 2A: http://www.rcreumatologia.org/index.php/rcr/rt/suppFiles/365/0; i: múltiples mediciones de autoclinimetría; j: múltiples mediciones objetivas realizadas por el observador. 
Figura 2.

Diagrama de Bosque (forest plot) de los estudios metanalizados: correlación entre conteo articular inflamatorio (observador $v s$. paciente).

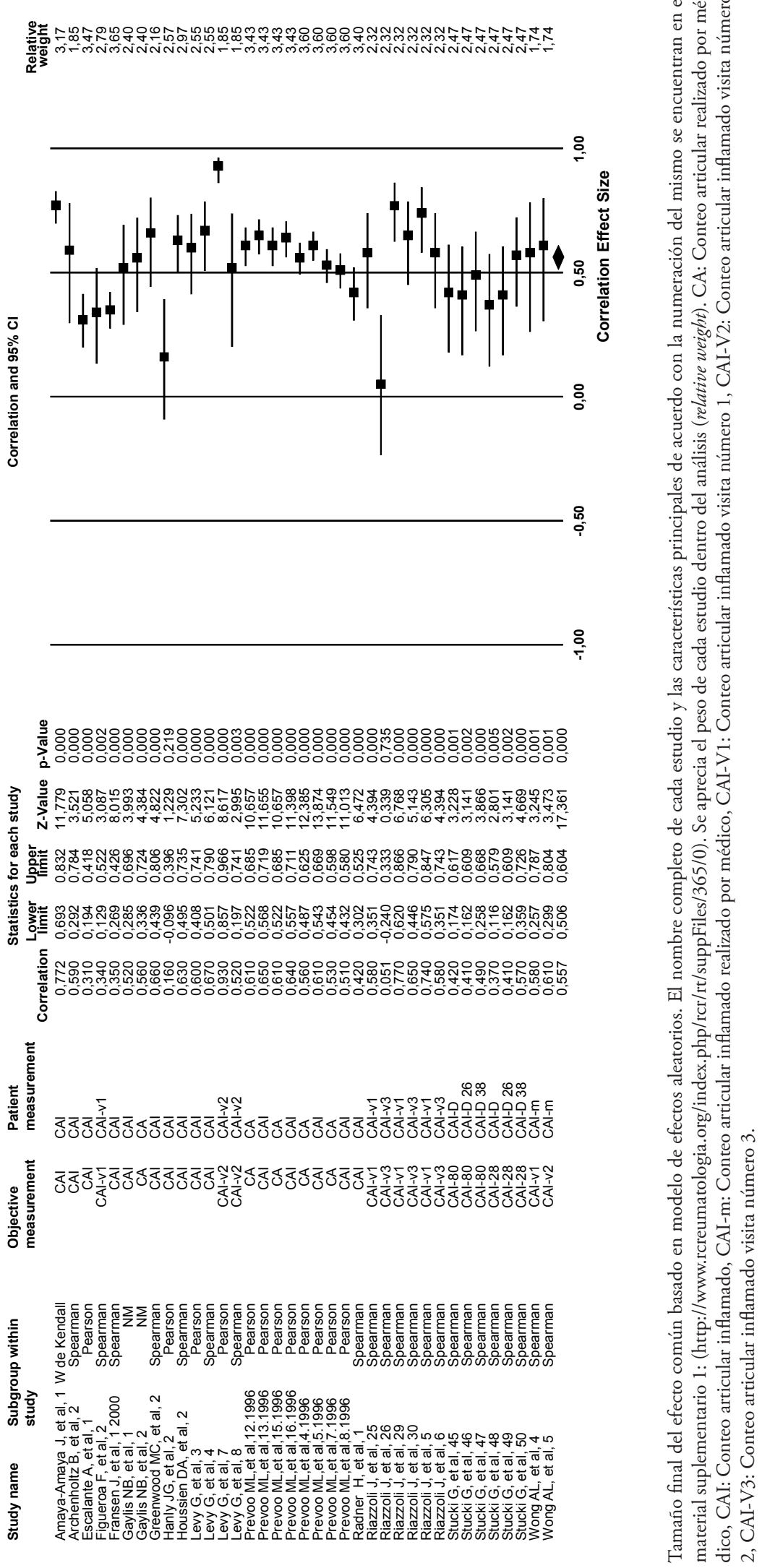



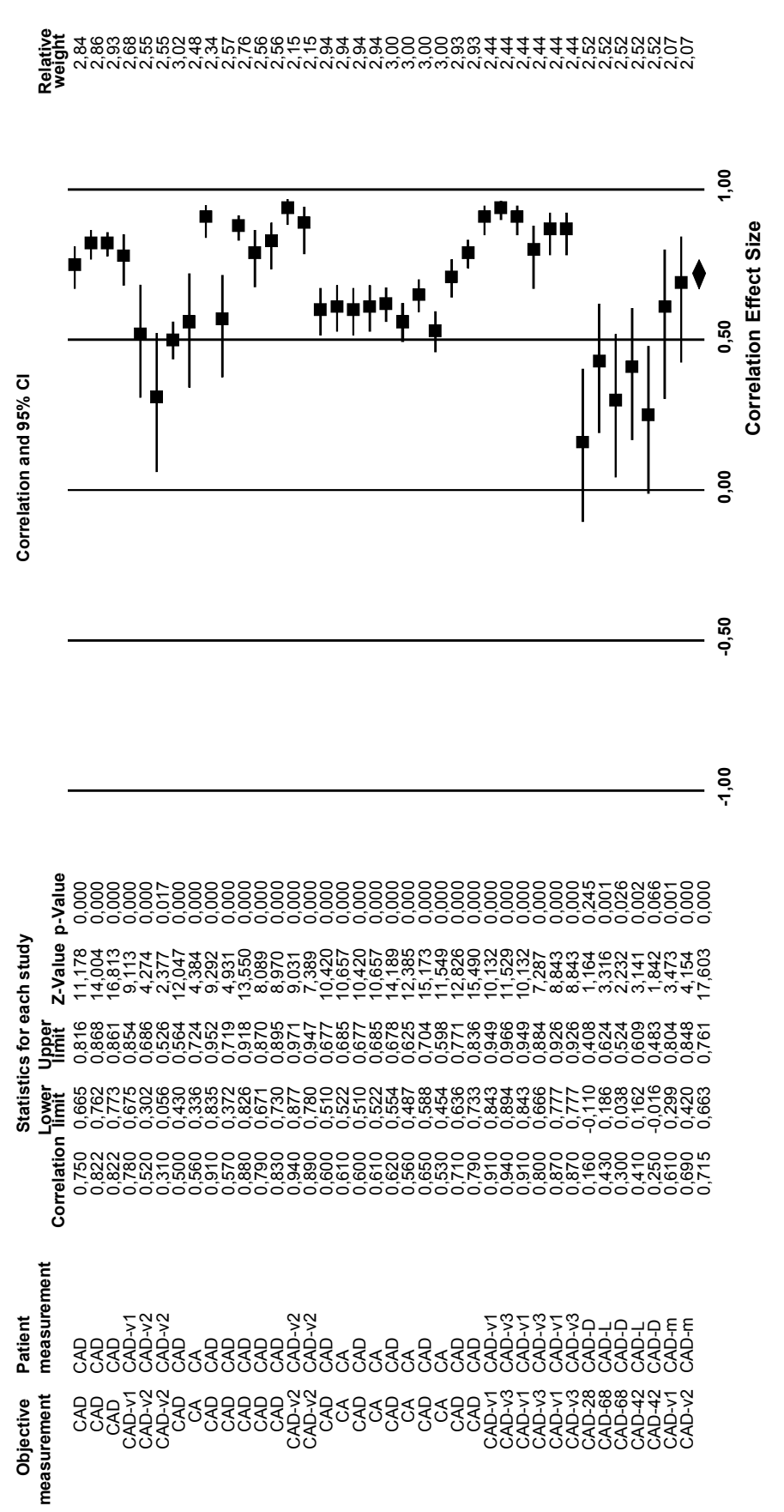

Figura 3. entre conteo articular doloroso (observador vs. paciente).
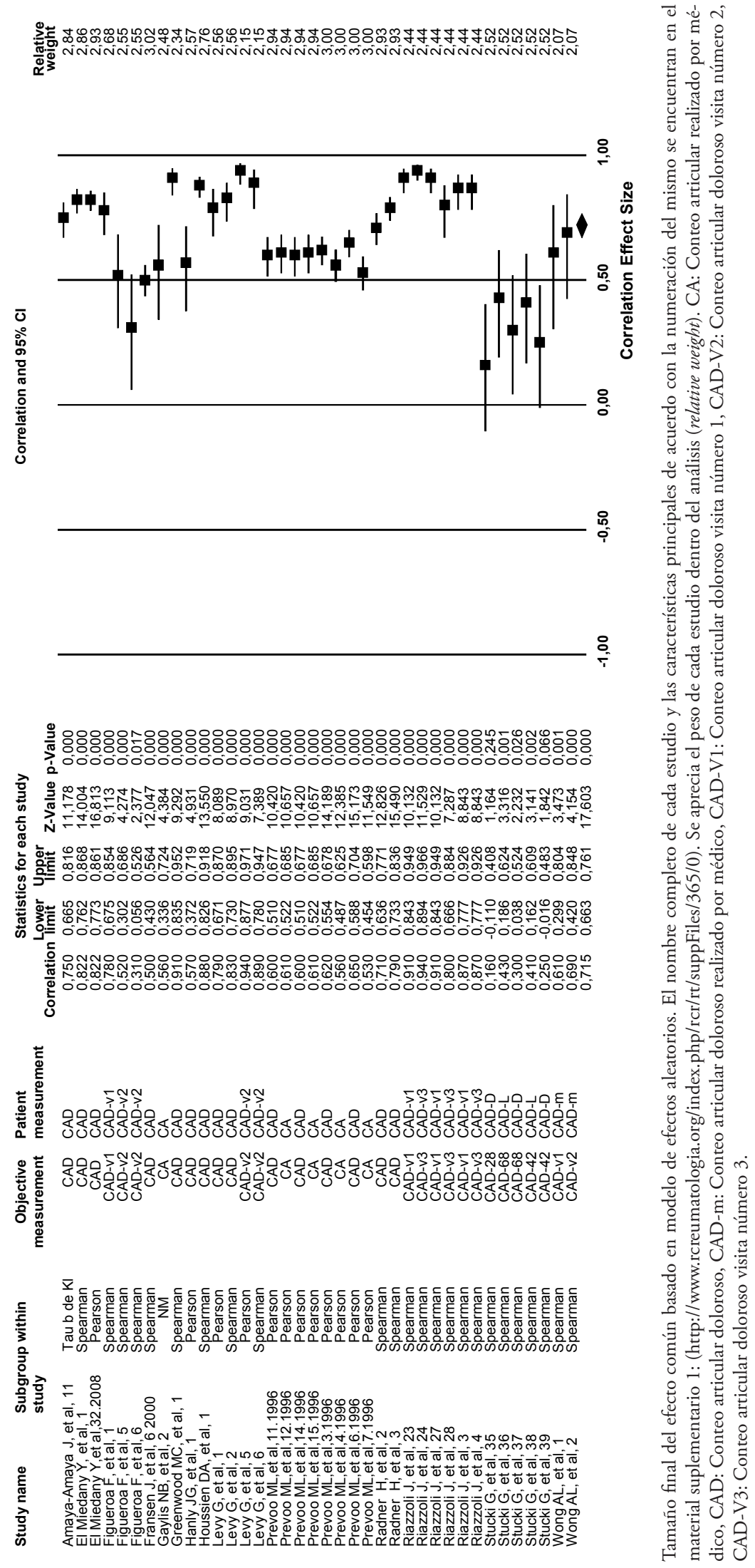


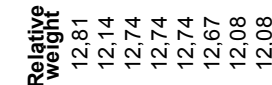
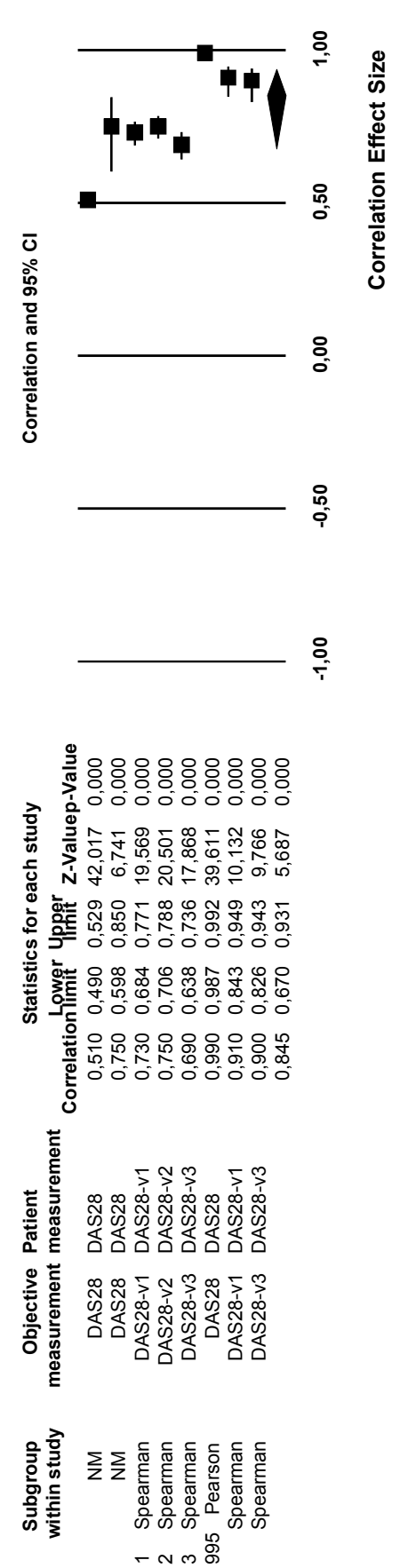

Figura 4.

Diagrama de Bosque (forest plot) de los estudios metanalizados: correlación entre el DAS28 evaluado por el observador y por el paciente.

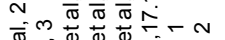

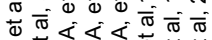

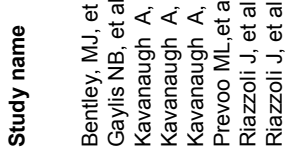

8

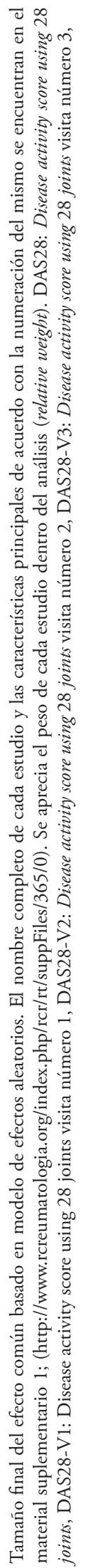


fico de embudo de cada análisis. Adicionalmente, allí se encuentran los metanálisis realizados comparando grupos de acuerdo con el estadístico utilizado para el cálculo de la correlación. En ninguno de los análisis los resultados se alteraron después de realizar el análisis de sensibilidad, excluyendo un estudio a la vez. En el análisis de correlación de CAI vs. CAI y DAS28 vs. múltiples mediciones, se encontró que el subgrupo de artículos analizados por medio del estadístico Pearson tuvo una correlación ligeramente mayor que la de los otros subgrupos (material suplementario), en los cuales también fue significativa; mientras que para el análisis de CAD vs. CAD en el subgrupo analizado por medio del estadístico Spearman, la correlación fue levemente superior. La correlación de CAI vs. CAI en los tres seguimientos fue muy similar mientras que en el análisis de CAD vs. CAD fue ligeramente superior en la tercera medición.

Para el segundo tamaño final del efecto basado en concordancias se desarrollaron 10 metanálisis, los cuales mostraron una concordancia significativa entre las medidas objetivas realizadas por el observador y aquellas de autoclinimetría desarrolladas por el paciente, con diferentes grados. En la tabla 2 se encuentran los resultados mostrando el tamaño final del efecto basado en el modelo de efectos aleatorios con el respectivo IC 95\%. Las figuras 5 y 6 muestran los diagramas de bosque correspondientes a análisis de concordancia entre el CAI y el CAD, respectivamente. Todos los análisis adicionales (comparación entre grupos según estadístico, análisis de sensibilidad y gráfico de embudo) se muestran en el material suplementario 2B.En el análisis de concordancias por subgrupos acordes al estadístico utilizado, se encontró que para CAI vs. CAI, DAS28 vs. DAS28 y CAD vs. CAD el subgrupo que utilizó el ICC tuvo una concordancia ligeramente superior a la de los grupos que utilizaron otro estadístico, siendo en éstos igualmente significativa.

Por medio del análisis utilizando el gráfico de embudo y la regresión de asimetría de Egger $(\mathrm{p}<0.05)$ se evidenció sesgo de publicación en 6 de los análisis de correlaciones y 4 de concordancias (Tablas 1 y 2); por lo anterior, y según lo planteado en la metodología, se realizaron análisis adicionales para corroborarlo. La prueba de análisis de fallas (classic fail-safe) indicó que se necesitaría que estuvieran perdidos de la búsqueda (o no incluidos) más de 1.000 estudios en el presente metanálisis para tener un valor $\mathrm{p}>$ alfa (muy poco probable en todos los que hubo necesidad de calcularlo, ver material suplementario $2 \mathrm{~A}$ y $2 \mathrm{~B}$ ). La prueba de correlación de rangos de Begg y Mazumdar fue no significativa (valor $\mathrm{p}$ a dos colas) y la prueba de ajuste de "corte y relleno" (trim and fill) no sugirió un riesgo menor que el originalmente calculado en la mayoría de ellos. Teniendo en cuenta todos estos análisis se consideró que el impacto del sesgo de publicación en estos metanálisis fue trivial. Por medio del análisis acumulativo (última columna en los diagramas de bosque), se muestran los estudios con mayor peso dentro del metanálisis en las figuras 2 a 6 y en el ma-

\begin{tabular}{|c|c|c|c|c|c|c|c|c|}
\hline & $\begin{array}{l}\text { Medición } \\
\text { objetiva }^{a}\end{array}$ & $\begin{array}{l}\text { Medición } \\
\text { autoclinimetría }\end{array}$ & $\begin{array}{l}\text { Número } \\
\text { de estudios } \\
\text { (subgrupos) }\end{array}$ & $\begin{array}{l}\text { Tamańo } \\
\text { del efecto } \\
\text { final b }\end{array}$ & IC 95\% & Valor $\mathbf{p}$ & $I^{2}$ & $\begin{array}{l}\text { Valor p } \\
\text { Egger }\end{array}$ \\
\hline & CAI & CAI & $8(18)$ & 0.437 & $0.374-0.497$ & $<0.0001$ & 60 & 0.21 \\
\hline & $\mathrm{CAI}^{\mathrm{C}}$ & CAI & $10(83)$ & 0.342 & $0.308-0.375$ & $<0.0001$ & 85 & $0.00016^{\mathrm{d}}$ \\
\hline & $\mathrm{CAI}^{\mathrm{e}}$ & CAIe & $7(14)$ & 0.424 & $0.327-0.512$ & $<0.0001$ & 66 & 0.30 \\
\hline Tabla 2. & CAD & CAD & $9(18)$ & 0.695 & $0.632-0.749$ & $<0.0001$ & 80 & $0.009^{\mathrm{d}}$ \\
\hline Resultados & $\mathrm{CAD}^{\mathrm{c}}$ & CAD & $10(66)$ & 0.534 & $0.500-0.566$ & $<0.0001$ & 90 & $0.0005^{\mathrm{d}}$ \\
\hline de los & $\mathrm{CAD}^{\mathrm{e}}$ & $\mathrm{CAD}^{\mathrm{e}}$ & $9(16)$ & 0.673 & $0.593-0.74$ & $<0.0001$ & 80 & $0.021^{\mathrm{d}}$ \\
\hline metanálisis con & DAS28 & DAS28 & $4(10)$ & 0.765 & $0.686-0.826$ & $<0.0001$ & 95 & 0.61 \\
\hline $\begin{array}{l}\text { concordancia } \\
\text { como tamańo }\end{array}$ & CDAI & Múltiple $^{\mathrm{f}}$ & $5(11)$ & 0.428 & $0.348-0.501$ & $<0.0001$ & 83 & 0.61 \\
\hline $\begin{array}{r}\text { final del } \\
\text { efecto basados } \\
\text { en modelos } \\
\text { de efectos } \\
\text { aleatorios. }\end{array}$ & \multicolumn{8}{|c|}{$\begin{array}{l}\text { a: Medición realizada por observador objetivo del equipo médico; b: concordancia basado en modelo de efectos } \\
\text { aleatorios; c: incluyendo conteo de articulaciones específicas; d: pruebas complementarias (Trim and fill, Begg y } \\
\text { Mazumdar, classic fail-safe) detalladas en material suplementario 2B (http://www.rcreumatologia.org/index.php/ } \\
\text { rcr/rt/suppFiles/365/0); e: visita 1; f: múltiples mediciones de autoclinimetría. }\end{array}$} \\
\hline
\end{tabular}




\begin{tabular}{|c|c|c|c|c|}
\hline Study name & \multicolumn{2}{|c|}{$\begin{array}{l}\text { Subgroup } \\
\text { within study }\end{array}$} & \multirow{2}{*}{$\begin{array}{l}\text { Objective } \\
\text { measurement } \\
\text { CAl-md }\end{array}$} & \multirow{2}{*}{$\begin{array}{l}\text { Patient } \\
\text { measuremer } \\
\text { CAI }\end{array}$} \\
\hline Cheung PP,et & al,1.2009 & ICC & & \\
\hline Cheung PP,et & al,11.2009 & Kappa & CAl & CAl \\
\hline Cheung PP,et & al, 12.2009 & Kappa & CAl & CAl \\
\hline Cheung PP,et & al, 13.2010 & ICC & CAl-md & $\mathrm{CAl}$ \\
\hline Cheung PP,et & al, 14.2010 & ICC & CAl-e & CAl \\
\hline Cheung PP,et & $\mathrm{al}, 2.2009$ & ICC & CAl-e & CAl \\
\hline Cheung PP,et & al,21.2010 & Pabak & CAl-md & CAl \\
\hline Cheung PP,et & al, 22.2010 & Pabak & CAl-e & CAl \\
\hline Cheung PP,et & $\mathrm{al}, 3.2009$ & ICC & CAI-ECO & CAl \\
\hline Figueroa $\mathrm{F}$, et & & ICC & CAl- v2 & CAl- v2 \\
\hline Hanly JG, et & & ICC & CAl & CAl \\
\hline Kavanaugh A & et al, 10 & ICC & CAl-v2 & CAl-v2 \\
\hline Kavanaugh $A$ & et al, 11 & ICC & CAl-v3 & CAl-v3 \\
\hline Kavanaugh $A$ & et al, 9 & ICC & CAl-v1 & CAl-v1 \\
\hline Radner $\mathrm{H}$, et & & ICC & CAl-v1 & CAlv1 \\
\hline Radner $\mathrm{H}$, et & & ICC & CAl-v2 & CAl v2 \\
\hline Stucki $G$, et al & & ICC & CAl-38 & CAl \\
\hline Wong AL, et & & ICC & CAl & CAl-m \\
\hline
\end{tabular}

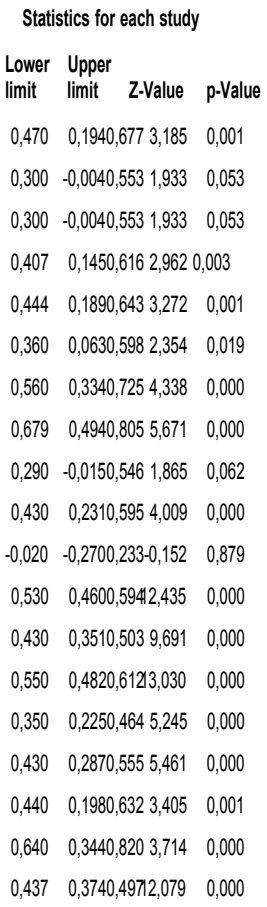

Concordance and $95 \% \mathrm{Cl}$

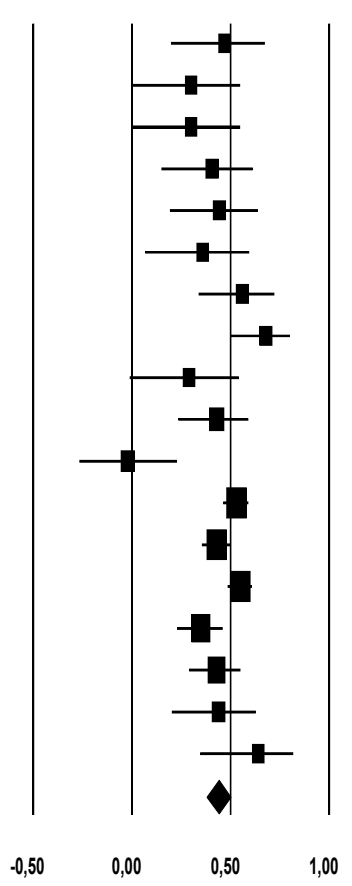

Concordance Effect Size

\section{Figura 5. \\ Diagrama de Bosque (forest plot) de los estudios metanalizados: concordancia entre conteo articular inflamatorio (observador $v s$ paciente).}

CA: Conteo articular realizado por médico, CAI: Conteo articular inflamado, CAI-e: Conteo articular inflamado realizado por enfermería, CAI-ECO: Conteo articular inflamado realizado por ecografía, CAI-md: Conteo articular inflamado realizado por médico, CAI-V1: Conteo articular inflamado visita número 1, CAI-V2: Conteo articular inflamado visita número 2, CAI-V3: Conteo articular inflamado visita número 3. Tamaño final del efecto común basado en modelo de efectos aleatorios. El nombre completo de cada estudio y las características principales de acuerdo con la numeración del mismo se encuentran en el material suplementario 1: http://www.rcreumatologia.org/index.php/rcr/rt/suppFiles/365/0. terial suplementario 2 (http://www.rcreumatologia. org/index.php/rcr/rt/suppFiles/365/0).

\section{Discusión}

Por medio del presente trabajo se demuestra el grado de correlación y concordancia entre las mediciones de autoclinimetría desarrolladas por pacientes con AR, y las herramientas de evaluación de la enfermedad y las medidas objetivas realizadas por el médico y personal de la salud entrenado, a través de revisión mundial sistemática de la literatura y metanálisis. De esta forma se encontró correlación y concordancia entre las medidas objetivas derivadas del médico y las medidas subjetivas aportadas por el paciente, destacando la correlación existente entre el CAI y el CAD, así como la encontrada entre índices de actividad de la AR, tales como DAS ${ }^{28}$ y SDAI y todas las variables medidas por el paciente. A pesar de que estas herramientas ya son ampliamente conocidas y utilizadas, pues le aportan al clínico información valiosa acerca del curso de la enfermedad y la existencia de signos de alarma, son más utilizadas en la investigación clínica ${ }^{19,30,88,134}$, que en la práctica cotidiana. Con los presentes resultados, se puede sugerir que más allá del interés investigativo ${ }^{31,41}$ la autoclinimetría debería ser aplicada rutinariamente. 


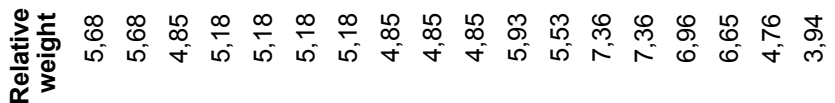

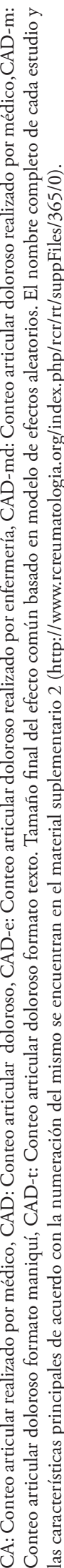

Bosque (forest plot) de los estudios metaanalizados: concordancia entre conteo articular doloroso (observador $v s$, paciente).

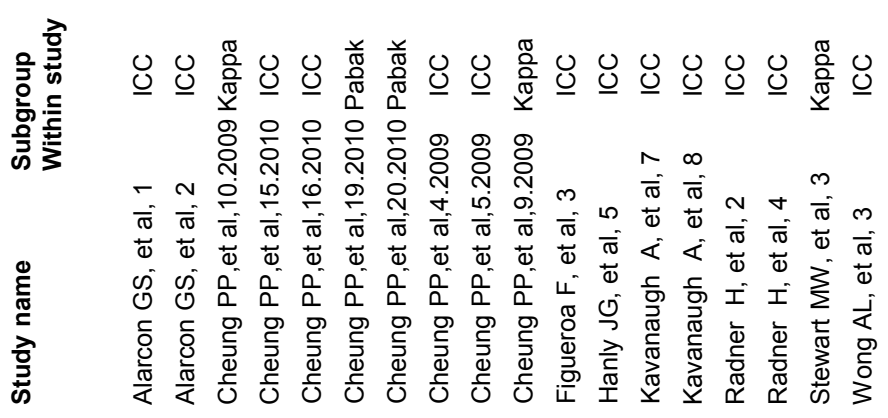

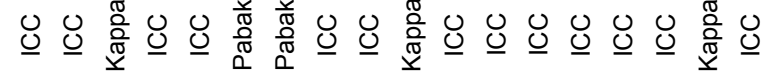

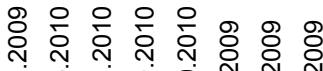

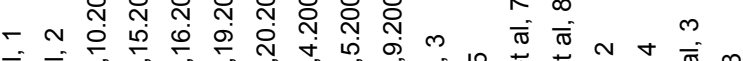

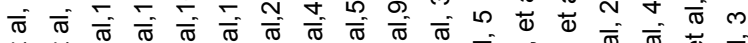

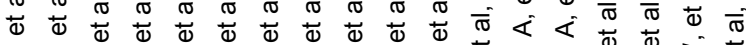

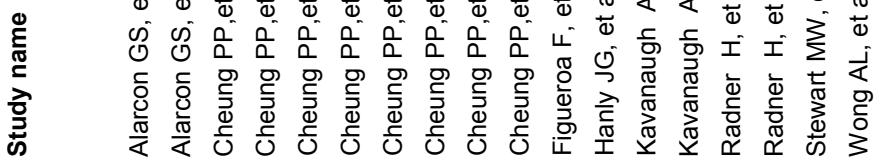

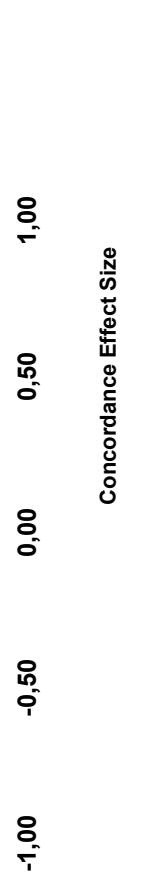

$\stackrel{8}{\frac{1}{5}}$

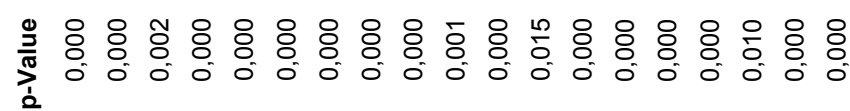

幽

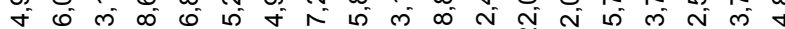

\%

र \&

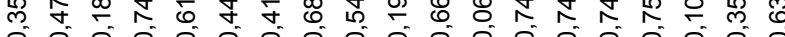

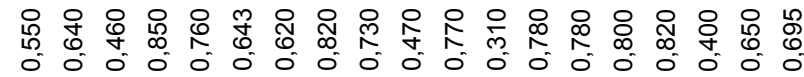

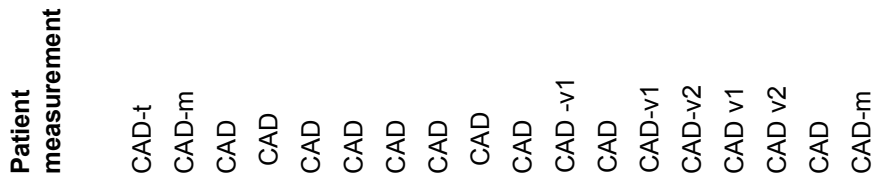

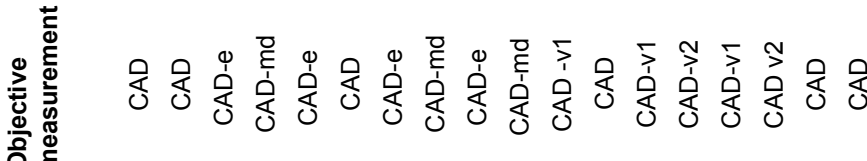


Estas herramientas son de fácil acceso e implementación, bajo costo y permiten, de forma adecuada y sencilla, realizar actividades de seguimiento objetivo en los pacientes con $\mathrm{AR}^{19,30,88,134}$. Adicionalmente, no solo tienen que ser accesibles por parte del reumatólogo, sino también por parte de médicos generales que laboran en clínicas especializadas en este campo ${ }^{19}$. Para cumplir dicho propósito, se han diseñado diferentes herramientas, que van más allá de un $\mathrm{CA}^{88}$, basadas en cuestionarios, escalas de medición de síntomas, resultados de paraclínicos, imágenes diagnósticas y conteos articulares cuantitativos, y que, a su vez, engloban adicionalmente la funcionalidad del paciente, la calidad de vida y el comportamiento del dolor percibido; por tanto, favorecen la adecuada toma de decisiones por parte del médico tratante al tiempo que aportan información pronóstico para cada paciente ${ }^{22,59,135}$.

La autoclinimetría puede ser aplicada en la sala de espera del consultorio ${ }^{135}$, haciendo de este tiempo un espacio productivo para la aplicación de estas herramientas, optimizando así el tiempo de consulta, de manera que el clínico pueda ejecutar un excelente examen físico, un adecuado conteo articular y, por supuesto, una adecuada aproximación terapéutica. La autoclinimetría engloba reportes que provienen directamente del paciente, sin necesidad de interpretación del médico, acerca de cómo se siente respecto a su funcionalidad, estado de salud, y tratamiento actual.

1. Aspectos generales de los instrumentos de desenlaces reportados por el paciente: La autoclinimetría es utilizada globalmente, y ha sido correlacionada significativamente con medidas objetivas utilizadas por el médico, no solo en el área de reumatología, como se demuestra en el presente estudio, sino también en otras enfermedades crónicas como: cáncer, asma, hipertensión, enfermedad cardíaca, accidente cerebrovascular, enfermedades psiquiátricas, migraña y diabetes ${ }^{25,136-140}$. Es importante resaltar que respecto al estado funcional, los cuestionarios diligenciados por los pacientes proveen medidas pronósticas de los desenlaces a largo plazo más importantes, como la funcionalidad, discapacidad laboral, costos de la enfermedad, necesidad de reemplazo articular quirúrgico y muerte prematura ${ }^{141,142}$.

Las medidas objetivas, realizadas por el equipo médico, tienen algunas limitaciones importantes, como lo es el tiempo necesario para computar e interpretar cada una de ellas; por ejemplo, el cálculo del DAS28PCR o DAS28-VSG requiere de una calculadora, computador o sitio web y el tiempo necesario para su cálculo es de 114 segundos, y el CDAI por su parte, toma 106 segundos en ser calculado $20,30,143-145$. Cada una de ellas requieren del conteo articular completo, el cual no es llevado a cabo por una gran cantidad de reumatólogos, adicionalmente cada una de ellas tiene puntos de corte diferentes en la interpretación de sus resultados. Estos dos hechos favorecen que algunos pierdan el interés en la utilización de estas medidas. Contrario a lo anterior, el RAPID3 del MDHAQ, una de las medidas de autoclinimetría más ampliamente utilizada, puede ser calculado en 5-10 segundos ${ }^{135}$. Otra gran desventaja es que por la premura del tiempo, las inquietudes de los pacientes y sus familiares no pueden ser resueltas ${ }^{136,146,147}$.

Debido a las dificultades y limitaciones antes mencionadas, los desenlaces reportados por los pacientes, han sido involucrados como herramientas para complementar las medidas objetivas realizadas por el clínico.

Adicionalmente, estos instrumentos son útiles para monitorizar al paciente a través del tiempo, debido a su validez, confiabilidad, factibilidad y sensibilidad al cambio. A su vez, se facilita la utilización de herramientas educativas, que mejoran la autoevaluación de la enfermedad, disminuyen la sensación de discapacidad y el riesgo de depresión, promueven el retorno a la actividad en sociedad y mejoran el apoyo social del paciente, la participación activa del paciente en su seguimiento a largo plazo, su adherencia al tratamiento beneficiando el control de la enfermedad y el pronóstico $^{137-139}$.

En los últimos años, el interés en la valoración del paciente con AR a partir de su propia perspectiva ha aumentado, al igual que el reconocimiento de la importancia de los desenlaces reportados por pacientes, gracias a las características antes mencionadas. Cabe resaltar que, gracias a la autoclinimetría, la historia clínica del paciente puede ser recolectada de tal forma que los datos allí contenidos puedan ser analizados de manera cuantitativa. Los datos extraídos de los cuestionarios son igual o más efectivos que ciertos análisis de laboratorio y el conteo articular formal para discriminar los pacientes que, estando en tratamiento, se encuentran con enfermedad activa, de aquellos que 
están en remisión, tanto en los ensayos clínicos como en la consulta externa ${ }^{28,147,148}$. Por ejemplo, se ha descrito que el marcador más significativo para predecir la mortalidad prematura a 5 años, en pacientes con AR, es el puntaje de capacidad funcional en las actividades de la vida diaria, adquirido mediante cuestionario, por encima de las pruebas de laboratorio, radiografías, u otros estudios imaginológicos ${ }^{31,149}$.

En un estudio realizado a pacientes vistos y evaluados extensivamente en 1973 y revalorados luego en 1982, las respuestas de los pacientes para llevar a cabo actividades de su vida cotidiana predijo más efectivamente la mortalidad a 5 años que todas las otras medidas clínicas conocidas; los pacientes que podían realizar $<80 \%$ de las actividades básicas cotidianas presentaron una supervivencia a 5 años del $50 \%$ que es proporcional a la supervivencia de los pacientes con Linfoma de Hodgkin en estadio IV y enfermedad coronaria de 3 vasos ${ }^{150,151}$. Similares hallazgos han sido reportados por Sokka y col. ${ }^{152}$, Callahan y col. ${ }^{151} \mathrm{y}$ Wolfe y col. ${ }^{153}$ quienes evaluaron el estado funcional mediante HAQ y MDHAQ.

2. Principales herramientas de autoclinimetría. El RAPID3 es un índice propuesto para la valoración y manejo de pacientes con $\mathrm{AR}$, que incluye 3 conjuntos de datos básicos del ACR sin tener en cuenta el CA formal: función física, EVA-D y EVA$\mathrm{G}$; puede ser calculado en 5-10 segundos en contraste con los 90-94 segundos que toma realizar un conteo de 28 articulaciones, 106 segundos para el CDAI y 114 segundos para el DAS28 $8^{82}$. Por otro lado, Pincus y col. ${ }^{41,83}$ demostraron el coeficiente de correlación de Spearman de 0.66 entre DAS28-VSG y RAPID3, 0.50 para DAS28-PCR y RAPID3 y 0.74 para CDAI y RAPID3, siendo todas estas correlaciones estadísticamente significativas $(\mathrm{p}<0.001)$. En contraste, Leeb y col. ${ }^{19,59}$ reportaron un grado de acuerdo sustancialmente menor entre RAPID 3 y DAS28 $r=0.32$, y entre RAPID3 y CDAI, $r=0.37$. En el presente metanálisis se encontró una adecuada correlación entre el DAS28 $y$ los RAPIDs ( $\mathrm{r}=0.62, \mathrm{p}<0.0001)$, al igual que en conjunto todas las medidas objetivas con los RAPID $3(\mathrm{r}=0.60, \mathrm{p}<0.0001)$.

De igual forma, las medidas de RAPID4 reflejan el estado clínico del paciente de manera similar al DAS28 y el CDAI ya que incluye el RAPID3 y el RADAI, que es un CA autodiligenciado,mundialmente validado. El RAPID4 puede ser calculado en aproximadamente 19 segundos ${ }^{154}$. Se ha encontrado acuerdo entre RAPID4 y CAD, DAS28, CDAI, SDAI pero no hubo correlación con el CAI. Esto puede ser debido a que este último conteo contribuye sólo al $17 \%$ del puntaje total del RAPID $4{ }^{155}$ y que el RADAI incluye únicamente el conteo de articulaciones dolorosas. De igual forma, se encontró, en el presente estudio, una correlación adecuada para el RAPID4 versus todas las medidas objetivas provenientes del médico o su equipo entrenado $(\mathrm{r}=0.62, \mathrm{p}<0.0001)$. Así mismo, el RAPID5 cursó con correlaciones altas $(\mathrm{r}=0.72$, $\mathrm{p}<0.0001)$, sin olvidar que éste último contiene una medida objetiva proveniente del médico.

El RAPID3, RAPID4 y RAPID5 dan resultados similares a los criterios de mejoría del ACR ya que pueden distinguir entre enfermedad activa y enfermedad controlada, en los pacientes con AR en estudios clínicos. Además, todos estos se correlacionan significativamente con el DAS2 $28,30,31,73$, como se demuestra también en este estudio $(\mathrm{r}=0.62, \mathrm{p}<0.0001)$.

Como se ha señalado, el CA es la medida más específica para evaluar la AR. Varios métodos de CA autorreportado han sido descritos desde los años ochenta, mostrando correlaciones de $\mathrm{r}=0.44-0.87$ con el CAD y CAI realizados por el médico ${ }^{29,42,73,117}$. Por ejemplo, el RADAI, (desarrollado por el paciente), tiene una correlación significativa con el CAD realizado por el médico ${ }^{29,101,126}$. Este mismo hallazgo fue demostrado en un estudio recientemente publicado de nuestro grupo $(\mathrm{r}=0.60, \mathrm{p}<0.001)^{45}$, así como en el presente metanálisis, donde todas las medidas evaluadas por el médico, incluidas en el estudio, se correlacionaron con el RADAI de una forma moderada $(r=0.57$, $\mathrm{p}<0.0001)$. Por otro lado, también se encontraron correlaciones moderadas a altas entre los CAD realizados por el médico y el paciente, así como para el CAI y el DAS28, cuando se realiza teniendo en cuenta el CA proveniente del paciente. Los valores de correlación encontrados fueron $\mathrm{r}=0.71, \mathrm{p}<0.0001 ; \mathrm{r}=0.57$, $\mathrm{p}<0.0001 ;$ y r $=0.84, \mathrm{p}<0.0001$, respectivamente. Cabe aclarar que estos índices no variaron mucho cuando se excluyó de los análisis el CA donde no se discrimina si corresponde al inflamatorio o doloroso.

El MDHAQ es una herramienta de autoclinimetría que incluye 6 actividades complejas de la vida diaria, las cuáles reflejan el estado actual del paciente ${ }^{143,156}$. Los reportes de HAQ y MDHAQ sugieren 
que los datos autodiligenciados son más confiables que los datos que pueden ser indagados por un profesional de la salud, y se correlacionan adecuadamente con los índices de actividad de la enfermedad como el DAS28, CDAI y SDAI $32,58,110,157,158$, lo que concuerda con lo encontrado en el presente trabajo (todas las medidas objetivas evaluadas versus el HAQ, $\mathrm{r}=0.44$, $\mathrm{p}<0.0001)$. La gran exactitud de estos datos puede ser explicada por el hecho de que la medida es tomada en un solo momento y por un solo observador, el paciente, en vez de dos observadores, el paciente y un profesional de la salud ${ }^{154}$.

El CDAI y el SDAI, por otro lado, son medidas que muestran correlación moderada a alta con todas las variables medidas por el paciente en el presente estudio, donde la correlación del SDAI y CDAI versus todas las medidas subjetivas estudiadas es de $\mathrm{r}=0.61 \mathrm{y}$ $r=0.65$, respectivamente, ambos con una significancia estadística de $\mathrm{p}<0.0001$. Se destacan los resultados presentados por Rintelen y col. ${ }^{60}$ quienes encontraron correlación significativa entre los puntajes de SDAI y CDAI con la calificación de dolor dada por el paciente (SDAI: $\mathrm{r}=0.660, \mathrm{p}<0.001$; CDAI: $\mathrm{r}=0.671, \mathrm{p}<0.001$ ). El SDAI tuvo una alta correlación con el EVA-G $(r=0.72, p<0.001)$ en nuestra cohorte ${ }^{45}$ al igual a lo reportado por Leeb y col. ${ }^{58}$ en 2005.

A través del presente metanálisis se demostró que los valores de correlación entre las diferentes medidas no se modifican al excluir un estudio a la vez (prueba de sensibilidad), lo que indica que estos resultados son robustos y consistentes; a su vez, se demuestra que los valores de correlación (o concordancia) al analizar subgrupos de estudios de acuerdo con el estadístico utilizado para calcularlo, no difieren de manera significativa cuando se analizan como un solo grupo (ver material suplementario 2). Es de resaltar que se encontró una heterogeneidad importante a lo largo de los diferentes metanálisis (Tablas 1 y 2), lo cual es esperable, ya que se trata de diferentes poblaciones raciales evaluadas, en diferentes momentos de la enfermedad y bajo diferentes regímenes de tratamiento, por lo cual consideramos que la escogencia a priori del modelo de efectos aleatorios estuvo adecuadamente indicada. Es importante destacar que se encontró concordancia entre tres (CAI, CAD y DAS28) de las medidas más importantes para evaluar el estado de actividad de la AR (Tabla 2).
3. Ventajas y desventajas de los desenlaces reportados por los pacientes: Las correlaciones entre las medidas tomadas por el médico y las reportadas por el paciente muestran ventajas para el manejo y pronóstico de éstos últimos. Los desenlaces reportados por el paciente han demostrado asociarse con mayor significancia al estado clínico que las pruebas de laboratorio o las radiografías ${ }^{30}$ para predecir, como se mencionó antes, mortalidad prematura, costos, entre otros $^{64,81,159-161}$.

Otro beneficio de los desenlaces reportados por los pacientes en AR es la capacidad que tienen para distinguir la enfermedad activa al igual que el DAS28 y el CDAI. Los dos poseen una correlación significativa con el CA, VSG, puntajes radiográficos y son igual o más informativos que los criterios de remisión 20, 50, 70 del ACR o el DAS. Por lo tanto, el paciente puede ejercer su propio control a través del tiempo ${ }^{22,146}$. Además, permiten diferenciar entre casos y controles en estudios clínicos en fase III y la modificación del tratamiento en los grupos placebo ${ }^{22,83,162-164}$.

Basado en los reportes de autoclinimetría, el médico puede ajustar diferentes estrategias para monitorizar al paciente ya que estos puntajes se encuentran disponibles en una hoja de seguimiento, lo que permite comparar la última visita con las anteriores antes de evaluar al paciente. Otro factor importante es que estos cuestionarios y escalas son de bajo costo y fácil uso por los pacientes ${ }^{30,146}$ y el clínico necesita de menos tiempo para calcularlos (p. Ej. MDHAQ, RAPID), como se mencionó previamente ${ }^{84,88}$.

Los cuestionarios pueden ser distribuidos a todos los pacientes en cada visita, quienes completan los instrumentos que han sido validados a escala mundial, haciendo parte de la infraestructura de la consulta reumatológica ${ }^{154}$. Así, estos instrumentos ayudan al paciente a que se prepare para la consulta mientras se encuentra en la sala de espera; mientras también el médico lo hace y ahorra tiempo revisando la historia antes de ver al paciente ${ }^{165}$; posteriormente, realiza una anamnesis completa y dirigida anotando los síntomas positivos, y se revisa la historia clínica reciente para completar y mejorar la precisión de la misma $^{22,126,141,154,159}$.

Otra de sus ventajas es la mayor adherencia al tratamiento por parte del paciente ${ }^{7-9}$ ya que éste, al ser un agente activo en la consulta, mejora la percep- 
ción de la enfermedad, siente que el examen físico va más allá del conteo articular y que su calidad de vida y satisfacción frente al tratamiento están siendo también evaluadas ${ }^{9}$. Por otro lado, esta herramienta indica al reumatólogo cuándo el curso de la enfermedad está en detrimento, por ejemplo cuando se observa un alto grado de actividad o deterioro en la funcionalidad del paciente, para así poder realizar intervenciones oportunas ${ }^{10,11}$. Como se mencionó anteriormente, la autoclinimetría es una de las medidas clínicas pronósticas más significativas para los desenlaces importantes a largo plazo en la AR, como lo son el estado funcional, la discapacidad laboral, los costos, cirugías de reemplazo articular y muerte prematura $^{12,13}$, y es por esto que esta herramienta ha sido incluida en el cuidado estándar del paciente en muchos países ${ }^{12,13,15}$.

A pesar de los beneficios anotados, la mayoría de las visitas de los pacientes con $\mathrm{AR}$ al reumatólogo no incluyen ni el CA ni el diligenciamiento de los cuestionarios $^{134}$. Esta situación puede deberse a las limitaciones que presentan los instrumentos, dónde, cerca de $20 \%$ de los pacientes necesitan ayuda para completar los cuestionarios ${ }^{166}$. Por otra parte, se observa el efecto suelo, donde por ejemplo, algunos pacientes presentan puntajes de HAQ normales pero sienten cierta limitación funcional ${ }^{32}$. En otros casos el médico no verifica el estado clínico diligenciado en los cuestionarios, por lo que los pacientes se sienten insatisfechos, después de haber completado los instrumentos y que el médico no los haya tenido en cuenta en su evaluación ${ }^{32}$.

En algunas ocasiones los instrumentos no son específicos, por ejemplo el HAQ, y otras medidas pueden mostrar mejoría en el estado general del paciente debido a otras situaciones no relacionadas con la AR, permitiendo, tal vez, una inadecuada aproximación terapéutica. Además, están sujetos a diferencias culturales, por ejemplo, los puntajes de dolor son más altos en los pacientes latinoamericanos y más bajos en los asiáticos ${ }^{167,168}$; deben ser traducidos y validados en varios idiomas y pueden ser alterados por ciertos pacientes que deseen respuestas diferentes ${ }^{22,146,161}$. Sin embargo, la experiencia a escala mundial ha mostrado que dicho fenómeno no es tan frecuente y las herramientas de autoclinimetría poseen una adecuada validez al compararlas con herramientas de clinimetría como el DAS28 $22,36,169$.
Otros autores han mostrado disparidades entre las medidas tomadas por el médico y por el paciente. Studenic y col. ${ }^{168}$ encontraron que los pacientes y los médicos frecuentemente difieren en la percepción de la actividad de la enfermedad, cuantificada por EVAG del paciente y la calificación dada por el médico. Esto pudo explicarse debido a que respecto al dolor, el paciente tuvo una peor percepción que el médico, mientras que en el CAI, la peor percepción fue la del médico. Estas dos discrepancias explican el 65\% de la discordancia entre las medidas tomadas por el médico y las reportadas por el paciente. En el presente metanálisis se demostró una correlación moderada entre las medidas por parte del médico y su equipo, evaluadas como grupo, y la EVA-G ( $r=0.54$, $\mathrm{p}<0.0001)$.

\section{Conclusiones}

A través de este estudio se puede concluir que las herramientas para los desenlaces reportados por los pacientes o autoclinimetría tienen una correlación y concordancia adecuadas con las medidas objetivas realizadas por el médico y su equipo entrenado, pueden ser administradas en la práctica clínica cotidiana y en ensayos clínicos. Consideramos que su uso puede ser ventajoso en nuestro medio ya que los cuestionarios son simples, estandarizados e idénticos para todos los pacientes, con preguntas sencillas y diligenciables en corto tiempo ${ }^{23,156}$, que a la vez incorporan la opinión de los mismos, convirtiéndolos en interlocutores activos de la consulta médica ${ }^{62,157}$.

Esta práctica puede aportar en el avance de la reumatología como especialidad y mejorar la calidad de vida de los pacientes con AR, debido a que la información puede ser recolectada fácil y rápidamente, en un tiempo limitado, en todas las consultas. Estos cuestionarios pueden ser diligenciados por los pacientes, sin importar el género, nivel educativo, edad o duración de la enfermedad. En consecuencia, ésta podría facilitar y optimizar el poco tiempo para su ejecución, dado el autoconocimiento de su enfermedad. $\mathrm{Al}$ ser ejecutada antes que el paciente sea examinado por el reumatólogo, la autoclinimetría podría ser útil en el Sistema de Seguridad Social Colombiano, ya que permite manejar un lenguaje similar entre examinado y examinador, al tiempo que brinda un panorama objetivo del estado de la enfermedad ${ }^{170}$, haciendo más eficiente el tiempo destinado a las consultas. 
Cabe aclarar que estas herramientas no pretenden sustituir los puntajes objetivos determinados durante la consulta, en otras palabras, la autoclinimetría no reemplaza el criterio clínico o el examen físico riguroso; por el contrario, complementa estas prácticas. Juntos, actúan en sinergismo y permiten al médico y al paciente alcanzar un consenso en la evaluación del estado de la enfermedad para mejorar, a largo plazo, la condición del paciente mediante el mejoramiento de su terapia.

Por medio del presente estudio pretendemos estimular a los clínicos a implementar estas medidas en la evaluación de pacientes con $\mathrm{AR}$, ya que están estandarizadas, son efectivas y eficientes. Estas parecen estar bien adaptadas a la mejoría continua de la calidad del cuidado del paciente con AR, contribuyendo a la obtención de datos entorno al estado funcional, dolor, estado global, fatiga y estado psicológico y que, muchas veces, no pueden ser obtenidos de otra forma. Esperamos que esto sea implementado en los centros de práctica de reumatología por todos los beneficios listados en el presente trabajo, mejorando la adherencia al tratamiento, reduciendo los costos y mejorando los desenlaces de esta patología.

\section{Abreviaturas}

ACR: American College of Rheumatology

AR: Artritis Reumatoide

AVD: Índices de realización de actividades diarias

BVS: Biblioteca Virtual de la Salud

CA: Conteo articular

CA-md: Conteo articular realizado por médico

CAD: Conteo articular doloroso

CAD-e: Conteo articular doloroso realizado por enfermería

CAD-md: Conteo articular doloroso realizado por médico

CAD-V1: Conteo articular doloroso visita número 1

CAD-V2: Conteo articular doloroso visita número 2

CAD-V3: Conteo articular doloroso visita número 3

CAI: Conteo articular inflamado

CAI-e: Conteo articular inflamado realizado por enfermería

CAI-ECO: Conteo articular inflamado realizado por ecografía
CAI-md: Conteo articular inflamado realizado por médico

CAI-V1: Conteo articular inflamado visita número 1

CAI-V2: Conteo articular inflamado visita número 2

CAI-V3: Conteo articular inflamado visita número 3

CDAI: Clinical disease activity index

CDAI-V1: Clinical disease activity index visita número 1

CDAI-V2: Clinical disease activity index visita número 2

CDAI-V3: Clinical disease activity index visita número 3

Clin28: Clinical remission assessed using 28 joints

CREA: Centro de estudio de enfermedades autoinmunes

DAS: Disease activity score

DAS28: Disease activity score using 28 joints

DAS28-e: Disease activity score using 28 joints realizado por enfermería

DAS28-md: Disease activity score using 28 joints realizado por médico

DAS28-ECO: Disease activity score using 28 joints realizado por ecografía

DAS28-V1: Disease activity score using 28 joints visita número 1

DAS28-V2: Disease activity score using 28 joints visita número 2

DAS28-V3: Disease activity score using 28 joints visita número 3

DASH Disabilities of Arm, Shoulder and Hand

DeCs: Descriptores en Ciencias de la Salud

EVA-D: Escala visual análoga dolor del paciente

EVA-D-V2: Escala visual análoga dolor del paciente visita número 2

EVA-G: Escala visual análoga global del paciente

EVA-G-V2: Escala visual análoga global del paciente visita número 2

GAT: Grip ability test

HAQ: Health assessment questionnaire

HAQ-DI: Health Assessment Questionnaire-Disability Index

HAQ-DI-V1: Health Assessment QuestionnaireDisability Index visita número 1

HAQ-DI-V2: Health Assessment Questionnaire-

Disability Index visita número 2

IC: Intervalo de confianza 
ID: Índice de dolor

IPQ-5: Illness Perception Questionnaire 5

MACTAR: McMaster Toronto Arthritis Patient Prefe-

rence Disability Questionnaire

MDHAQ: Medical health assessment questionnaire

$\mathrm{MeSH}$ : Medical Subject Headings

NM: No mencionado

NS: No significativo

PCR: Proteína $\mathrm{C}$ reactiva

PF-10: Physical Functioning Scale -10

PG: Physician generated

PR: Patient reported

PRISMA: Preferred reporting items for systematic reviews and meta-analyses

PROs: Patient-reported outcomes

PS-AVD: Performance and Satisfaction of Activity of

Daily Living

RADAI: Rheumatoid arthritis disease activity index

RADAI-J: Rheumatoid arthritis disease activity index tender joints

RADAI-SF: Rheumatoid arthritis disease activity index short form

RADAI-TS: Rheumatoid arthritis disease activity index total score

RADAR: Rapid assessment disease activity in rheumatology

RADAR-m: Rapid assessment disease activity in rheumatology formato maniquí

RADAR-t: Rapid assessment disease activity in rheu- matology formato texto

RAI: Indice Articular de Richie

RAPID: Routine assessment of patients index data

RAPID3-A: Routine assessment of patients index data

3 en pacientes con alta severidad

RAPID3-B: Routine assessment of patients index data

3 en pacientes con baja severidad

RAPID3-M: Routine assessment of patients index data

3 en pacientes con moderada severidad

RAPID3-B: Routine assessment of patients index data

3 en pacientes cercanos a remisión

RAPID4: Routine assessment of patients index data 4

RAPID5: Routine assessment of patients index data 5

RAPS: Rheumatoid Arthritis Pain Scale

RAQoL: Rheumatoid arthritis quality of life

SciELO: Scientific Electronic Library Online

SDAI: Simplified disease activity index

SDAI-V1: Simplified disease activity index visita número 1

SDAI-V2: Simplified disease activity index visita número 2

SDAI-V3: Simplified disease activity index visita número 3

SFC: Clasificación funcional Steinbrocker

Thom: Índice articular de Thomson

VSG: Velocidad de eritrosedimentación

Las siglas corresponden a aquellas incluidas en el artículo y en el material suplementario 1 y 2 (Ay B).

\section{Referencias}

1. Scott DL, Wolfe F, Huizinga TW. Rheumatoid arthritis. Lancet [Internet]. Elsevier Ltd; 2010 Sep 25 [cited 2011 Jun 11];376(9746):1094-108. Available from: http://www.ncbi. nlm.nih.gov/pubmed/20870100.

2. Van Riel PL, Fransen J. Established rheumatoid arthritis: clinical assessments. Best practice \& research. Clinical rheumatology [Internet]. 2007 Oct [cited 2012 Mar 31];21(5):807-25. Available from: http://www.ncbi.nlm. nih.gov/pubmed/17870029.

3. Rindfleisch JA, Muller D. Diagnosis and management of rheumatoid arthritis. American family physician [Internet]. 2005 Sep 15 [cited 2012 Jul 4];72(6):1037-47. Available from: http://www.ncbi.nlm.nih.gov/pubmed/16190501.

4. Anaya J-M. Severe rheumatoid valvular heart disease. Clinical rheumatology [Internet]. 2006 Sep [cited 2012 Jul
5];25(5):743-5. Available from: http://www.ncbi.nlm.nih. gov/pubmed/16249828.

5. DeMaria AN. Relative risk of cardiovascular events in patients with rheumatoid arthritis. The American journal of cardiology [Internet]. 2002 Mar 21;89(6A):33D-38D. Available from: http://www.ncbi.nlm.nih.gov/pubmed/11909559.

6. Sokka T, Krishnan E, Häkkinen A, HP. Functional disability in rheumatoid arthritis patients compared with a community population in Finland. Arthritis and rheumatism [Internet]. 2003 Jan [cited 2011 Aug 18];48(1):59-63. Available from: http://www.ncbi.nlm.nih.gov/pubmed/12528104.

7. Wolfe F. A reappraisal of HAQ disability in rheumatoid arthritis. Arthritis and rheumatism [Internet]. 2000 Dec;43(12):2751-61. Available from: http://www.ncbi.nlm. nih.gov/pubmed/11145033.

8. Cadena J, Vinaccia S, Pérez A, Rico MI, Hinojosa R, Anaya J-M. The impact of disease activity on the quality 
of life, mental health status, and family dysfunction in colombian patients with rheumatoid arthritis. Journal of clinical rheumatology : practical reports on rheumatic \& musculoskeletal diseases [Internet]. 2003 Jun [cited 2012 Jul 5];9(3):142-50. Available from: http://www.ncbi.nlm. nih.gov/pubmed/17041449.

9. Rojas-Villarraga A, Bayona J, Zuluaga N, Mejía S, Hincapié M-E, Anaya J-M. The impact of rheumatoid foot on disability in Colombian patients with rheumatoid arthritis. BMC musculoskeletal disorders [Internet]. 2009 Jan [cited 2012 Jul 5];10:67. Available from: http://www.pubmedcentral.nih. gov/articlerender.fcgi?artid=2702313\&tool=pmcentrez\&ren dertype $=$ abstract.

10. Sandoo A, Carroll D, Metsios GS, Kitas GD, Veldhuijzen van Zanten JJ. The association between microvascular and macrovascular endothelial function in patients with rheumatoid arthritis: a cross-sectional study. Arthritis research $\&$ therapy [Internet]. BioMed Central Ltd; 2011 Jan [cited 2011 Dec 13];13(3):R99. Available from: http://www.pubmedcentral. nih.gov/articlerender.fcgi? artid=3218914\&tool=pmcentrez $\&$ rendertype $=$ abstract

11. Peters MJ, Symmons DPM, McCarey D, Dijkmans B a C, Nicola P, Kvien TK, et al. EULAR evidence-based recommendations for cardiovascular risk management in patients with rheumatoid arthritis and other forms of inflammatory arthritis. Annals of the rheumatic diseases [Internet]. 2010 Feb [cited 2012 Dec 24];69(2):325-31. Available from: http://www.ncbi.nlm.nih.gov/pubmed/19773290.

12. Delgado-Vega AM, Anaya J-M. Meta-analysis of HLA-DRB1 polymorphism in Latin American patients with rheumatoid arthritis. Autoimmunity reviews [Internet]. 2007 Jun [cited 2012 Jan 20];6(6):402-8. Available from: http://www.ncbi. nlm.nih.gov/pubmed/17537386.

13. Alamanos Y, Drosos AA. Epidemiology of adult rheumatoid arthritis. Autoimmunity reviews [Internet]. 2005 Mar [cited 2012 Jun 29];4(3):130-6. Available from: http://www.ncbi. nlm.nih.gov/pubmed/15823498.

14. Symmons D, Turner G, Webb R, Asten P, Barrett E, Lunt M, et al. The prevalence of rheumatoid arthritis in the United Kingdom: new estimates for a new century. Rheumatology (Oxford, England) [Internet]. 2002 Jul [cited 2012 Jul 4];41(7):793-800. Available from: http://www.ncbi.nlm. nih.gov/pubmed/12096230.

15. Delgado-Vega AM, Martín J, Granados J, Anaya JM. [Genetic epidemiology of rheumatoid arthritis: what to expect from Latin America?]. Biomédica : revista del Instituto Nacional de Salud [Internet]. 2006 Dec [cited 2012 Apr 22];26(4):562-84. Available from: http://www.ncbi.nlm. nih.gov/pubmed/17315483.

16. Kvien TK, Uhlig T, Ødegård S, Heiberg MS. Epidemiological aspects of rheumatoid arthritis: the sex ratio. Annals of the New York Academy of Sciences [Internet]. 2006 Jun [cited 2012 Jul 4];1069:212-22. Available from: http://www.ncbi. nlm.nih.gov/pubmed/16855148.

17. Gabriel SE, Crowson CS, Kremers HM, Doran MF, Turesson C, O'Fallon WM, et al. Survival in rheumatoid arthritis: a population-based analysis of trends over 40 years. Arthri- tis and rheumatism [Internet]. 2003 Jan [cited 2012 Jul 4];48(1):54-8. Available from: http://www.ncbi.nlm.nih. gov/pubmed/12528103.

18. Schneeberger EE, Marengo MF, Papasidero SB, Chaparrodel Moral RE CG. Clinimetría en artritis reumatoidea. Rev Argent Reumatol. 2008;2(19).

19. Leeb BF, Sautner J, Mai HTH, Haindl PM, Deutsch C, Rintelen B. A comparison of patient questionnaires and composite indexes in routine care of rheumatoid arthritis patients. Joint, bone, spine [Internet]. 2009 Dec [cited 2012 Jan 20];76(6):658-64. Available from: http://www.ncbi.nlm. nih.gov/pubmed/19520595.

20. Pincus T. Pain, function, and RAPID scores: vital signs in chronic diseases, analogous to pulse and temperature in acute diseases and blood pressure and cholesterol in longterm health. Bulletin of the NYU hospital for joint diseases [Internet]. 2008 Jan [cited 2012 Apr 22];66(2):155-65. Available from: http://www.ncbi.nlm.nih.gov/pubmed/18537789.

21. Pincus T, Sokka T. Quantitative clinical assessment in busy rheumatology settings: the value of short patient questionnaires. The Journal of rheumatology [Internet]. 2008 Jul [cited 2012 Apr 23];35(7):1235-7. Available from: http://www. ncbi.nlm.nih.gov/pubmed/18609730.

22. Pincus T, Yazici Y, Bergman MJ. Patient questionnaires in rheumatoid arthritis: advantages and limitations as a quantitative, standardized scientific medical history. Rheumatic diseases clinics of North America [Internet]. 2009 Nov [cited 2011 Aug 18];35(4):735-43, vii. Available from: http://www. ncbi.nlm.nih.gov/pubmed/19962618.

23. Pincus T, Swearingen CJ. The HAQ compared with the MDHAQ: "keep it simple, stupid" (KISS), with feasibility and clinical value as primary criteria for patient questionnaires in usual clinical care. Rheumatic diseases clinics of North America [Internet]. 2009 Nov [cited 2011 Aug 18];35(4):787-98, ix. Available from: http://www.ncbi.nlm. nih.gov/pubmed/19962623.

24. Pincus T, Sokka T. Complexities in the quantitative assessment of patients with rheumatic diseases in clinical trials and clinical care. Clinical and experimental rheumatology [Internet]. 2005;23(5 Suppl 39):S1-9. Available from: http:// www.ncbi.nlm.nih.gov/pubmed/16273778.

25. Lasch KE, Marquis P, Vigneux M, Abetz L, Arnould B, Bayliss $\mathrm{M}$, et al. PRO development: rigorous qualitative research as the crucial foundation. Quality of life research : an international journal of quality of life aspects of treatment, care and rehabilitation [Internet]. 2010 Oct [cited 2012 Jul 8];19(8):1087-96. Available from: http://www.pubmedcentral.nih.gov/articlerender.fcgi?artid=2940042\&tool=pmcent rez\&rendertype $=$ abstract.

26. Bruce B, Fries JF. The Health Assessment Questionnaire (HAQ). Clinical and experimental rheumatology [Internet]. [cited 2012 Apr 17];23(5 Suppl 39):S14-8. Available from: http://www.ncbi.nlm.nih.gov/pubmed/16273780.

27. Seror R, Tubach F, Baron G, Guillemin F, Ravaud P. Measure of function in rheumatoid arthritis : individualized or classical scales? Arthritis \& Rheumatism. 2010. 
28. Her M, Kavanaugh A. Patient-reported outcomes in rheumatoid arthritis. Current opinion in rheumatology [Internet]. 2012 May [cited 2012 Apr 23];24(3):327-34. Available from: http://www.ncbi.nlm.nih.gov/pubmed/22410543.

29. Stucki G, Liang MH, Stucki S, Brühlmann P, Michel BA. A self-administered rheumatoid arthritis disease activity index (RADAI) for epidemiologic research. Psychometric properties and correlation with parameters of disease activity. Arthritis and rheumatism [Internet]. 1995 Jun [cited 2012 Apr 20];38(6):795-8. Available from: http://www.ncbi.nlm. nih.gov/pubmed/7779122.

30. Pincus T. Are patient questionnaire scores as "scientific" as laboratory tests for rheumatology clinical care? Bulletin of the NYU hospital for joint diseases [Internet]. 2010 Jan [cited 2012 Jul 8];68(2):130-9. Available from: http://www.ncbi. nlm.nih.gov/pubmed/20632989.

31. Salaffi F, Stancati A, Neri R, Grassi W, Bombardieri S. Measuring functional disability in early rheumatoid arthritis: the validity, reliability and responsiveness of the Recent-Onset Arthritis Disability (ROAD) index. Clinical and experimental rheumatology [Internet]. 2005 [cited 2012 Apr 25];23(5 Suppl 39):S31-42. Available from: http://www.ncbi.nlm. nih.gov/pubmed/16273783.

32. Pincus T, Yazici Y, Bergman M. Development of a multidimensional health assessment questionnaire (MDHAQ) for the infrastructure of standard clinical care. Clinical and experimental rheumatology [Internet]. [cited 2012 Apr 24];23(5 Suppl 39):S19-28. Available from: http://www. ncbi.nlm.nih.gov/pubmed/16273781.

33. Li T, Wells G, Westhovens R, Tugwell P. Validation of a simple activity participation measure for rheumatoid arthritis clinical trials. Rheumatology (Oxford) [Internet]. 2009 Feb [cited 2012 Feb 19];48(2):170-5. Available from: http://www.ncbi. nlm.nih.gov/pubmed/19141573.

34. Wolfe F, Michaud K, Pincus T. Development and validation of the health assessment questionnaire II: a revised version of the health assessment questionnaire. Arthritis and rheumatism [Internet]. 2004 Oct [cited 2012 Jan 20];50(10):3296-305. Available from: http://www.ncbi.nlm. nih.gov/pubmed/15476213.

35. Citera G, Arriola MS, Maldonado-Cocco JA, Rosemffet MG, Sánchez MM, Goñi MA, et al. Validation and cross cultural adaptation of an argentine spanish version of the health assessment questionnaire disability index. Journal of clinical rheumatology : practical reports on rheumatic \& musculoskeletal diseases [Internet]. 2004 Jun [cited 2012 Apr 15];10(3):110-5. Available from: http://www.ncbi.nlm. nih.gov/pubmed/17043481.

36. Rojas-LLorena GA, Posada-Coello A, Gilbert-Toledano M, Hernández-Martínez A, González IR, Fernández-Madero I, Taylor-Jiménez B T-MR. Validación de la versión cubana de la dimensión física del Cuestionario de Evaluación de Salud (HAQ) en cubanos con Artritis Reumatoide. (CU-HAQ). Rev Cub Reumatol. 2002;4(1):43-55.

37. Pincus T, Sokka T, Kavanaugh A. Quantitative documentation of benefit/risk of new therapies for rheumatoid arthritis: patient questionnaires as an optimal measure in standard care.
Clinical and experimental rheumatology [Internet]. 2004 [cited 2012 Apr 25];22(5 Suppl 35):S26-33. Available from: http://www.ncbi.nlm.nih.gov/pubmed/15552511.

38. Bruce B, Fries JF. The Stanford Health Assessment Questionnaire: a review of its history, issues, progress, and documentation. The Journal of rheumatology [Internet]. 2003 Jan [cited 2012 Apr 24];30(1):167-78. Available from: http:// www.ncbi.nlm.nih.gov/pubmed/12508408.

39. Caballero-Uribe C V. Artritis reumatoide como enfermedad de alto costo. Rev Col Reumatol. 2004;11(3):225-31.

40. De Jong Z, Van der Heijde D, McKenna SP, Whalley D. The reliability and construct validity of the RAQoL: a rheumatoid arthritis-specific quality of life instrument. British journal of rheumatology [Internet]. 1997 Aug [cited 2012 Apr 24];36(8):878-83. Available from: http://www.ncbi.nlm. nih.gov/pubmed/9291857.

41. Pincus T, Swearingen CJ, Bergman MJ, Colglazier CL, Kaell AT, Kunath AM, et al. RAPID3 (Routine Assessment of Patient Index Data) on an MDHAQ (Multidimensional Health Assessment Questionnaire): agreement with DAS28 (Disease Activity Score) and CDAI (Clinical Disease Activity Index) activity categories, scored in five versus more than. Arthritis care \& research [Internet]. 2010 Feb [cited 2011 Jul 22];62(2):181-9. Available from: http://www.ncbi.nlm. nih.gov/pubmed/20191516.

42. Mason JH, Anderson JJ, Meenan RF, Haralson KM, LewisStevens D, Kaine JL. The rapid assessment of disease activity in rheumatology (radar) questionnaire. Validity and sensitivity to change of a patient self-report measure of joint count and clinical status. Arthritis and rheumatism [Internet]. 1992 Feb [cited 2012 Mar 9];35(2):156-62. Available from: http:// www.ncbi.nlm.nih.gov/pubmed/1734905.

43. Stewart MW, Palmer DG, Knight RG. A self-report articular index measure of arthritic activity: investigations of reliability, validity and sensitivity. The Journal of rheumatology [Internet]. 1990 Aug [cited 2012 Jul 15];17(8):1011-5. Available from: http://www.ncbi.nlm.nih.gov/pubmed/2213775.

44. Stucki G, Stucki S, Brühlmann P, Maus S, Michel BA. Comparison of the validity and reliability of self-reported articular indices. British journal of rheumatology [Internet]. 1995 Aug [cited 2012 Apr 22];34(8):760-6. Available from: http://www.ncbi.nlm.nih.gov/pubmed/7551662.

45. Amaya-Amaya Jenny, Botello-Corzo Diana, Calixto Omar-Javier, Calderón-Rojas Rolando, Domínguez AuraMaría, Cruz-Tapias Paola, Montoya-Ortiz Gladys, Mantilla Rubén-Darío, Anaya Juan-Manuel R-VA, et al. Usefulness of patients-reported outcomes in rheumatoid arthritis focus group. Arthritis [Internet]. 2012 Jan [cited 2012 Nov 2];In Press.935187. Available from: http://www.pubmedcentral. nih.gov/articlerender.fcgi?artid=3465872\&tool=pmcentrez \&rendertype $=$ abstract.

46. Vermeer M. Is DAS28 Remission Good Enough? Disease Activity and Functionality in Rheumatoid Arthritis, Results of the DREAM Remission Induction Cohort. [abstract]. Arthritis Rheum. 2010;62(suppl 10):314.

47. Hanly JG, Mosher D, Sutton E, Weerasinghe S, Theriault D. Self-assessment of disease activity by patients with rheumatoid 
arthritis. The Journal of rheumatology [Internet]. $1996 \mathrm{Sep}$ [cited 2012 Jul 15];23(9):1531-8. Available from: http:// www.ncbi.nlm.nih.gov/pubmed/8877920.

48. Gøtzsche PC. Reporting of outcomes in arthritis trials measured on ordinal and interval scales is inadequate in relation to meta-analysis. Annals of the rheumatic diseases [Internet]. 2001 Apr [cited 2013 Feb 4];60(4):349-52. Available from: http://www.pubmedcentral.nih.gov/articlerender.fcgi?artid= 1753597\&tool=pmcentrez\&rendertype=abstract.

49. Barton JL, Criswell LA, Kaiser R, Chen Y, Schillinger D. Systematic review and metaanalysis of patient self-report versus trained assessor joint counts in rheumatoid arthritis. The Journal of rheumatology [Internet]. 2009 Dec [cited 2012 Apr 15];36(12):2635-41. Available from: http://www. pubmedcentral.nih.gov/articlerender.fcgi? artid $=2874463 \& \mathrm{t}$ ool=pmcentrez\&rendertype=abstract.

50. Kalyoncu U, Dougados M, Daurès J-P, Gossec L. Reporting of patient-reported outcomes in recent trials in rheumatoid arthritis: a systematic literature review. Annals of the rheumatic diseases [Internet]. 2009 Feb [cited 2013 Feb 4];68(2):183-90. Available from: http://www.ncbi.nlm.nih. gov/pubmed/18375533.

51. Liberati A, Altman DG, Tetzlaff J, Mulrow C, Gøtzsche PC, Ioannidis JPA, et al. The PRISMA statement for reporting systematic reviews and meta-analyses of studies that evaluate health care interventions: explanation and elaboration. Journal of clinical epidemiology. 2009 Oct;62(10):e1-34.

52. Van der Leeden M, Steultjens MPM, Terwee CB, Rosenbaum $\mathrm{D}$, Turner D, Woodburn J, et al. A systematic review of instruments measuring foot function, foot pain, and foot-related disability in patients with rheumatoid arthritis. Arthritis and rheumatism [Internet]. 2008 Sep 15 [cited 2012 Jan 20];59(9):1257-69. Available from: http://www.ncbi.nlm. nih.gov/pubmed/18759256.

53. Aletaha D, Smolen J. The Simplified Disease Activity Index (SDAI) and the Clinical Disease Activity Index (CDAI): a review of their usefulness and validity in rheumatoid arthritis. Clinical and experimental rheumatology [Internet]. 2005 [cited 2012 Apr 24];23(5 Suppl 39):S100-8. Available from: http://www.ncbi.nlm.nih.gov/pubmed/16273793.

54. Williamson A, Hoggart B. Pain: a review of three commonly used pain rating scales. Journal of clinical nursing [Internet]. 2005 Aug;14(7):798-804. Available from: http://www.ncbi. nlm.nih.gov/pubmed/16000093.

55. OCEBM Levels of Evidence Working Group. The Oxford 2011 Levels of Evidence. Oxford Centre for Evidence-Based Medicine. http://www.cebm.net/index.aspx?o=5653. 2011.

56. Smolen JS, Breedveld FC, Schiff MH, Kalden JR, Emery P, Eberl G, et al. A simplified disease activity index for rheumatoid arthritis for use in clinical practice. Rheumatology (Oxford, England) [Internet]. 2003 Feb 1 [cited 2012 Jul 8];42(2):244-57. Available from: http://www.rheumatology. oupjournals.org/cgi/doi/10.1093/rheumatology/keg072.

57. Aletaha D, Nell VPK, Stamm T, Uffmann M, Pflugbeil S, Machold K, et al. Acute phase reactants add little to composite disease activity indices for rheumatoid arthritis: validation of a clinical activity score. Arthritis research \& therapy [Internet].
2005 Jan [cited 2011 Aug 10];7(4):R796-806. Available from: http://www.pubmedcentral.nih.gov/articlerender.fcg i?artid=1 175030\& tool=pmcentrez\&rendertype $=$ abstract.

58. Leeb BF, Andel I, Sautner J, Bogdan M, Maktari A, Nothnagl $\mathrm{T}$, et al. Disease activity measurement of rheumatoid arthritis: Comparison of the simplified disease activity index (SDAI) and the disease activity score including 28 joints (DAS28) in daily routine. Arthritis and rheumatism [Internet]. 2005 Feb 15 [cited 2012 Jan 20];53(1):56-60. Available from: http:// www.ncbi.nlm.nih.gov/pubmed/15696565.

59. Leeb BF, Haindl PM, Maktari A, Nothnagl T, Rintelen B. Patient-centered rheumatoid arthritis disease activity assessment by a modified RADAI. The Journal of rheumatology [Internet]. 2008 Jul [cited 2012 Jun 26];35(7):1294-9. Available from: http://www.ncbi.nlm.nih.gov/pubmed/18484698.

60. Rintelen B, Haindl PM, Maktari a, Nothnagl T, Hartl E, Leeb BF. SDAI/CDAI levels in rheumatoid arthritis patients are highly dependent on patient's pain perception and gender. Scandinavian journal of rheumatology [Internet]. 2008 [cited 2012 Jan 20];37(6):410-3. Available from: http://www.ncbi. nlm.nih.gov/pubmed/18759162.

61. Rintelen B, Haindl PM, Sautner J, Leeb B a BF, Deutsch C. The rheumatoid arthritis disease activity index- 5 in daily use. Proposal for disease activity categories. The Journal of rheumatology [Internet]. 2009 May [cited 2012 Jan 20];36(5):918-24. Available from: http://www.ncbi.nlm. nih.gov/pubmed/19332635.

62. Radner H. Clinical utility and validity of Self Assessed Joint Involvement in Rheumatoid Arthritis (RA) Patients with Low Active Disease. [abstract]. Arthritis and rheumatism. 2010;62(suppl 10):1745.

63. Radner H, Grisar J, Smolen JS, Stamm T, Aletaha D. Value of self-performed joint counts in rheumatoid arthritis patients near remission. Arthritis research \& therapy [Internet]. 2012 Jan [cited 2012 Nov 5];14(2):R61. Available from: http:// www.pubmedcentral.nih.gov/articlerender.fcgi? artid $=34464$ 29\&tool=pmcentrez\&rendertype $=$ abstract.

64. Werner C AM, Kaliski K S, Salazar Q K, Bustos M L, Rojas R MT, Baumert L C, et al. [Knowledge about their disease and treatment among patients with rheumatoid arthritis]. Revista médica de Chile [Internet]. 2006 Dec [cited 2012 Jul 8];134(12):1500-6. Available from: http://www.ncbi. nlm.nih.gov/pubmed/17277865.

65. Figueroa F, Braun-Moscovici Y, Khanna D, Voon E, Gallardo L, Luinstra D, et al. Patient self-administered joint tenderness counts in rheumatoid arthritis are reliable and responsive to changes in disease activity. The Journal of rheumatology [Internet]. 2007 Jan [cited 2012 Jul 15];34(1):54-6. Available from: http://www.ncbi.nlm.nih.gov/pubmed/17216678.

66. El Miedany Y, Youssef SS, El Gaafary M. Short-term outcome after anti-tumor necrosis factor-alpha therapy in rheumatoid arthritis: do we need to revise our assessment criteria? The Journal of rheumatology [Internet]. 2006 Mar [cited 2012 Nov 5];33(3):490-7. Available from: http://www.ncbi.nlm. nih.gov/pubmed/16511918.

67. El Miedany Y, Palmer D. Can standard rheumatology clinical practice be patient-based? British journal of nursing 
(Mark Allen Publishing) [Internet]. 2008 [cited 2012 Apr 22];17(10):673-5. Available from: http://www.ncbi.nlm.nih. gov/pubmed/18563011.

68. Esteve-Vives J, Batlle-Gualda E, Reig A. Spanish version of the Health Assessment Questionnaire: reliability, validity and transcultural equivalency. Grupo para la Adaptación del HAQ a la Población Española. The Journal of rheumatology [Internet]. 1993 Dec [cited 2012 Nov 5];20(12):2116-22. Available from: http://www.ncbi.nlm.nih.gov/pubmed/8014941.

69. Callahan LF, Brooks RH, Summey JA, Pincus T. Quantitative pain assessment for routine care of rheumatoid arthritis patients, using a pain scale based on activities of daily living and a visual analog pain scale. Arthritis and rheumatism [Internet]. 1987 Jun [cited 2012 Nov 5];30(6):630-6. Available from: http://www.ncbi.nlm.nih.gov/pubmed/3606681.

70. Pincus T, Callahan LF, Brooks RH, Fuchs HA, Olsen NJ, Kaye JJ. Self-report questionnaire scores in rheumatoid arthritis compared with traditional physical, radiographic, and laboratory measures. Annals of internal medicine [Internet]. 1989 Feb 15 [cited 2012 Apr 24];110(4):259-66. Available from: http://www.ncbi.nlm.nih.gov/pubmed/2913913.

71. Gaston-Johansson F, Gustafsson M. Rheumatoid arthritis: determination of pain characteristics and comparison of RAI and VAS in its measurement. Pain [Internet]. 1990 Apr [cited 2012 Nov 5];41(1):35-40. Available from: http://www.ncbi. nlm.nih.gov/pubmed/2352764.

72. Abraham N, Blackmon D, Jackson JR, Bradley LA, Lorish CD, Alarcón GS. Use of self-administered joint counts in the evaluation of rheumatoid arthritis patients. Arthritis care and research : the official journal of the Arthritis Health Professions Association [Internet]. 1993 Jun [cited 2012 Jul 15];6(2):78-81. Available from: http://www.ncbi.nlm.nih. gov/pubmed/8399430.

73. Escalante A. What do self-administered joint counts tell us about patients with rheumatoid arthritis? Arthritis care and research : the official journal of the Arthritis Health Professions Association [Internet]. 1998 Aug [cited 2012 Apr 24];11(4):280-90. Available from: http://www.ncbi.nlm.nih. gov/pubmed/9791327.

74. Alarcón GS, Tilley BC, Li S, Fowler SE, Pillemer SR. Selfadministered joint counts and standard joint counts in the assessment of rheumatoid arthritis. MIRA Trial Group. Minocycline in RA. The Journal of rheumatology [Internet]. 1999 May [cited 2012 Jul 15];26(5):1065-7. Available from: http://www.ncbi.nlm.nih.gov/pubmed/10332969.

75. Calvo FA, Calvo A, Berrocal A, Pevez C, Romero F, Vega E, et al. Self-administered joint counts in rheumatoid arthritis: comparison with standard joint counts. The Journal of rheumatology [Internet]. 1999 Mar [cited 2012 Jul 15];26(3):536-9. Available from: http://www.ncbi.nlm.nih. gov/pubmed/10090158.

76. Wong AL, Wong WK, Harker J, Sterz M, Bulpitt K, Park G, et al. Patient self-report tender and swollen joint counts in early rheumatoid arthritis. Western Consortium of Practicing Rheumatologists. The Journal of rheumatology [Internet]. 1999 Dec [cited 2012 Apr 23];26(12):2551-61. Available from: http://www.ncbi.nlm.nih.gov/pubmed/10606362.
77. Anderson DL. Development of an instrument to measure pain in rheumatoid arthritis: Rheumatoid Arthritis Pain Scale (RAPS). Arthritis and rheumatism [Internet]. 2001 Aug [cited 2012 Nov 2];45(4):317-23. Available from: http:// www.ncbi.nlm.nih.gov/pubmed/11501718.

78. Dwyer KA, Coty MB, Smith CA, Dulemba S, Wallston KA. A comparison of two methods of assessing disease activity in the joints. Nursing research [Internet]. [cited $2012 \mathrm{Nov}$ 2];50(4):214-21. Available from: http://www.ncbi.nlm.nih. gov/pubmed/11480530.

79. Pincus T, Yazici Y, Bergman M, Swearingen C, Harrington T. A proposed approach to recognize "near-remission" quantitatively without formal joint counts or laboratory tests: a patient self-report questionnaire routine assessment of patient index data (RAPID) score as a guide to a "continuous quality improvement" s. Clinical and experimental rheumatology [Internet]. [cited 2012 Apr 24];24(6 Suppl 43):S-60-5; quiz S-66-73. Available from: http://www.ncbi.nlm.nih. gov/pubmed/17083765.

80. Carter JD, Lodhi AB, Nagda SR, Ricca L, Ward C, Traina $\mathrm{E}$, et al. Determining rheumatologists' accuracy at assessing functional disability in rheumatoid arthritis patients using the Health Assessment Questionnaire-Disability Index. The Journal of rheumatology [Internet]. 2007 May [cited 2012 Nov 2];34(5):958-63. Available from: http://www.ncbi.nlm. nih.gov/pubmed/17407221.

81. Levy G, Cheetham C, Cheatwood A, Burchette R. Validation of patient-reported joint counts in rheumatoid arthritis and the role of training. The Journal of rheumatology [Internet]. 2007 Jun [cited 2012 Jun 26];34(6):1261-5. Available from: http://www.ncbi.nlm.nih.gov/pubmed/17444590.

82. Pincus T, Swearingen CJ, Bergman M, Yazici Y. RAPID3 (Routine Assessment of Patient Index Data 3), a rheumatoid arthritis index without formal joint counts for routine care: proposed severity categories compared to disease activity score and clinical disease activity index categories. The Journal of rheumatology [Internet]. 2008 Nov 15 [cited 2012 Jun 26];35(11):2136-47. Available from: http://www.jrheum. com/subscribers/08/11/2136.html.

83. Pincus T, Bergman MJ, Yazici Y, Hines P, Raghupathi K, Maclean R. An index of only patient-reported outcome measures, routine assessment of patient index data 3 (RAPID3), in two abatacept clinical trials: similar results to disease activity score (DAS28) and other RAPID indices that include physician-reported measures. Rheumatology (Oxford, England) [Internet]. 2008 Mar [cited 2011 Aug 18];47(3):345-9. Available from: http://www.ncbi.nlm.nih. gov/pubmed/18238788.

84. Shaver TS, Anderson JD, Weidensaul DN, Shahouri SH, Shahouri SS, Busch RE, et al. The problem of rheumatoid arthritis disease activity and remission in clinical practice. The Journal of rheumatology [Internet]. 2008 Jun [cited 2012 Jun 26];35(6):1015-22. Available from: http://www. ncbi.nlm.nih.gov/pubmed/18412311.

85. Kern M. The accuracy of self-administered rheumatoid arthritis activity compared with clinician exam. Arthritis and Rheumatism. 2009;60. 
86. Khan NA, Yazici Y, Calvo-Alen J, Dadoniene J, Gossec L, Hansen TM, et al. Reevaluation of the role of duration of morning stiffness in the assessment of rheumatoid arthritis activity. The Journal of rheumatology [Internet]. 2009 Nov [cited 2012 Nov 2];36(11):2435-42. Available from: http:// www.ncbi.nlm.nih.gov/pubmed/19833759.

87. Li X. Validation of rheumatoid arthritis disease activity index (RADAI) in a North American cohort of patients with early rheumatoid arthritis (RA). Arthritis and Rheumatism. 2009;60:965.

88. Wolfe F, Michaud K. Proposed metrics for the determination of rheumatoid arthritis outcome and treatment success and failure. The Journal of rheumatology. 2009 Jan;36(1):27-33.

89. Bentley MJ, Greenberg JD, Reed GW. A modified rheumatoid arthritis disease activity score without acute-phase reactants (mDAS28) for epidemiological research. The Journal of rheumatology [Internet]. 2010 Aug 1 [cited 2012 Jan 20];37(8):1607-14. Available from: http://www.ncbi.nlm. nih.gov/pubmed/20595282.

90. Kavanaugh A, Lee SJ, Weng HH, Chon Y, Huang X-Y, Lin S-L. Patient-derived joint counts are a potential alternative for determining Disease Activity Score. The Journal of rheumatology [Internet]. 2010 May [cited 2012 Nov 2];37(5):1035-41. Available from: http://www.ncbi.nlm. nih.gov/pubmed/20156946.

91. Gaylis NB. Computer Assisted Patient Reported (PR) versus Physician Generated (PG) Joint Count (JC) and DAS28 Scores for Rheumatoid Arthritis (RA) Patients. [abstract]. Arthritis Rheum. 2010;62(supple 10):1747.

92. Bergman M. Routine Assessment Of Patient Index Data-3 (RAPID3), A Patient-Reported Index To Guide A Treat-ToTarget Strategy For Rheumatoid Arthritis In Usual Care. Arthritis and Rheumatism. 2011;63(10).

93. Lupash Daniel, Patel Aarat M, Amity Christine L, Frydrych Lynne M, Sippel Derek, Jones Donald M. et al; Comparison Of The Patient-Based Routine Assessment Of Patient Index 3 In Usual Care Of Rheumatoid Arthritis To The PhysicianBased Disease Activity Score-28 Joint Count And Clinical Disease Activity Index. Arthritis Rheum. 2011;63(Suppl 10):2143.

94. Weinblatt ME. Patient-Reported Disease Activity Including Joint Assessment: A Comparison of RADAI (Rheumatoid Arthritis Disease Activity Index) and RAPID3 (Routine Assessment of Patient Index Data 3) in Patients Treated with Certolizumab Pegol Over 12 Weeks. [abstract]. Arthritis Rheum. 2011;63(suppl 10):1230.

95. Karpouzas GA, Dolatabadi S, Moran R, Li N, Nicassio PM, Weisman MH. Correlates and predictors of disability in vulnerable US Hispanics with rheumatoid arthritis. Arthritis care \& research [Internet]. 2012 Sep [cited $2012 \mathrm{Nov}$ 2];64(9):1274-81. Available from: http://www.ncbi.nlm.nih. gov/pubmed/22489073.

96. Castrejón I, Yazici Y, Pincus T. Patient self-report RADAI joint counts on a MDHAQ are informative in a cohort of consecutive patients with rheumatic diseases other than RA seen in usual care. Arthritis care $\&$ research [Internet]. 2012 Jul 17 [cited 2012 Nov 5]; Available from: http://www.ncbi. nlm.nih.gov/pubmed/22807473.
97. Tammaru M, Singh G, Hanson E, Maimets K. The adaptation, reliability and validity testing of the Estonian version of the Health Assessment Questionnaire's Disability Index. Rheumatology international [Internet]. 2007 Nov [cited 2012 Oct 15];28(1):51-9. Available from: http://www.ncbi. nlm.nih.gov/pubmed/17641894.

98. Sokka T, Hetland ML, Mäkinen H, Kautiainen H, HørslevPetersen K, Luukkainen RK, et al. Remission and rheumatoid arthritis: data on patients receiving usual care in twenty-four countries. Arthritis and rheumatism [Internet]. 2008 Sep [cited 2012 Oct 15];58(9):2642-51. Available from: http:// www.ncbi.nlm.nih.gov/pubmed/18759292.

99. Cheung P. How reliably can rheumatoid arthritis patients calculate DAS28? A comparison study with ultrasonography, physician and nurse. Arthritis and Rheumatism. 2009;60.

100. Cheung PP, Ruyssen-Witrand A, Gossec L, Paternotte S, Le Bourlout C, Mazieres M, et al. Reliability of patient self-evaluation of swollen and tender joints in rheumatoid arthritis: A comparison study with ultrasonography, physician, and nurse assessments. Arthritis care $\&$ research [Internet]. 2010 Aug [cited 2012 Nov 2];62(8):1112-9. Available from: http://www.ncbi.nlm.nih.gov/pub$\mathrm{med} / 20235213$.

101. Bossert M, Prati C, Vidal C, Bongain S, Toussirot E, Wendling D. Evaluation of self-report questionnaires for assessing rheumatoid arthritis activity: a cross-sectional study of RAPID3 and RADAI5 and flare detection in 200 patients. Joint, bone, spine : revue du rheumatism [Internet]. 2012 Jan [cited 2012 Jul 16];79(1):57-62. Available from: http://www.ncbi.nlm.nih.gov/pubmed/21550283.

102. Bijlsma JW, Huiskes CJ, Kraaimaat FW, Vanderveen MJ, Huber-Bruning O. Relation between patients' own health assessment and clinical and laboratory findings in rheumatoid arthritis. The Journal of rheumatology [Internet]. 1991 May [cited 2012 Nov 5];18(5):650-3. Available from: http://www.ncbi.nlm.nih.gov/pubmed/1865409.

103. Prevoo ML, Van 't Hof MA, Kuper HH, Van Leeuwen MA, Van de Putte LB, Van Riel PL. Modified disease activity scores that include twenty-eight-joint counts. Development and validation in a prospective longitudinal study of patients with rheumatoid arthritis. Arthritis and rheumatism [Internet]. 1995 Jan [cited 2012 Apr 24];38(1):44-8. Available from: http://www.ncbi.nlm. nih.gov/pubmed/7818570.

104. Prevoo ML, Kuper IH, van't Hof MA, Van Leeuwen MA, Van de Putte LB, Van Riel PL. Validity and reproducibility of self-administered joint counts. A prospective longitudinal follow up study in patients with rheumatoid arthritis. The Journal of rheumatology [Internet]. 1996 May [cited 2012 Feb 23];23(5):841-5. Available from: http://www. ncbi.nlm.nih.gov/pubmed/8724295.

105. Taal E, Abdel-Nasser AM, Rasker JJ, Wiegman O. A selfreport Thompson articular index: what does it measure? Clinical rheumatology [Internet]. 1998 Jan [cited 2012 Jul 15];17(2):125-9. Available from: http://www.ncbi. nlm.nih.gov/pubmed/9641509.

106. Tijhuis GJ, De Jong Z, Zwinderman AH, Zuijderduin WM, Jansen LM, Hazes JM, et al. The validity of the 
Rheumatoid Arthritis Quality of Life (RAQoL) questionnaire. Rheumatology (Oxford, England) [Internet]. 2001 Oct [cited 2012 Apr 24];40(10):1112-9. Available from: http://www.ncbi.nlm.nih.gov/pubmed/11600740.

107. Kievit W, Welsing PMJ, Adang EMM, Eijsbouts a M, Krabbe PFM, Van Riel PLCM. Comment on the use of self-reporting instruments to assess patients with rheumatoid arthritis: the longitudinal association between the DAS28 and the VAS general health. Arthritis and rheumatism [Internet]. 2006 Oct 15 [cited 2012 Jan 20];55(5):745-50. Available from: http://www.ncbi.nlm. nih.gov/pubmed/17013821.

108. Van der Heijde D, Klareskog L, Singh A, Tornero J, MeloGomes J, Codreanu C, et al. Patient reported outcomes in a trial of combination therapy with etanercept and methotrexate for rheumatoid arthritis: the TEMPO trial. Annals of the rheumatic diseases [Internet]. $2006 \mathrm{Mar}$ [cited 2012 Nov 5];65(3):328-34. Available from: http:// www.pubmedcentral.nih.gov/articlerender.fcgi?artid=179 8055\&tool=pmcentrez\&rendertype $=$ abstract.

109. Veehof MM, Ten Klooster PM, Taal E, Van Riel PLCM, Van de Laar MAFJ. Psychometric properties of the Rheumatoid Arthritis Disease Activity Index (RADAI) in a cohort of consecutive Dutch patients with RA starting anti-tumor necrosis factor treatment. Annals of the rheumatic diseases [Internet]. 2008 Jun [cited 2012 Oct 18];67(6):789-93. Available from: http://www.ncbi.nlm. nih.gov/pubmed/17974597.

110. Salaffi F, Cimmino MA, Leardini G, Gasparini S, Grassi W. Disease activity assessment of rheumatoid arthritis in daily practice: validity, internal consistency, reliability and congruency of the Disease Activity Score including 28 joints (DAS28) compared with the Clinical Disease Activity Index (CDAI). Clinical and experimental rheumatology [Internet]. 2009 [cited 2012 Jul 8];27(4):552-9. Available from: http://www.ncbi.nlm.nih.gov/pubmed/19772784.

111. Smedstad LM, Kvien TK, Moum T, Vaglum P. Correlates of patients' global assessment of arthritis impact. A 2-year study of 216 patients with RA. Scandinavian journal of rheumatology [Internet]. 1997 Jan [cited 2012 Oct 15];26(4):259-65. Available from: http://www.ncbi.nlm. nih.gov/pubmed/9310104.

112. Abourazzak FE, Benbouazza K, Amine B, Bahiri R, Lazrak N, Bzami F, et al. Psychometric evaluation of a Moroccan version of health assessment questionnaire for use in Moroccan patients with rheumatoid arthritis. Rheumatology international [Internet]. 2008 Oct [cited 2012 Oct 9];28(12):1197-203. Available from: http://www.ncbi. nlm.nih.gov/pubmed/18535829.

113. Uhlig T, Haavardsholm EA, Kvien TK. Comparison of the Health Assessment Questionnaire (HAQ) and the modified HAQ (MHAQ) in patients with rheumatoid arthritis. Rheumatology (Oxford, England) [Internet]. 2006 Apr [cited 2012 Oct 15];45(4):454-8. Available from: http://www.ncbi.nlm.nih.gov/pubmed/16287925.

114. Uhlig T, Kvien TK, Pincus T. Test-retest reliability of disease activity core set measures and indices in rheumatoid arthritis. Annals of the rheumatic diseases [Internet]. 2009
Jun [cited 2012 Oct 16];68(6):972-5. Available from: http://www.ncbi.nlm.nih.gov/pubmed/18957489.

115. Jankowska B. Clinical and sociodemographic factors determining quality of life in rheumatoid arthritis (RA). Family Medicine and Primary Care Review. 2010;12(4).

116. Hewlett SE, Haynes J, Shepstone L, Kirwan JR. Rheumatoid arthritis patients cannot accurately report signs of inflammatory activity. British journal of rheumatology [Internet]. 1995 Jun [cited 2012 Apr 23];34(6):547-53. Available from: http://www.ncbi.nlm.nih.gov/pub$\mathrm{med} / 7633798$.

117. Houssien DA, Stucki G, Scott DL. A patient-derived disease activity score can substitute for a physician-derived disease activity score in clinical research. Rheumatology (Oxford, England) [Internet]. 1999 Jan [cited 2012 Jul 8];38(1):48-52. Available from: http://www.ncbi.nlm. nih.gov/pubmed/10334682.

118. Adams J, Burridge J, Mullee M, Hammond A, Cooper C. Correlation between upper limb functional ability and structural hand impairment in an early rheumatoid population. Clinical rehabilitation [Internet]. 2004 Jun [cited 2012 Nov 2];18(4):405-13. Available from: http:// www.ncbi.nlm.nih.gov/pubmed/15180124.

119. Greenwood MC, Hakim AJ, Carson E, Doyle D V. Touchscreen computer systems in the rheumatology clinic offer a reliable and user-friendly means of collecting quality-of-life and outcome data from patients with rheumatoid arthritis. Rheumatology (Oxford, England) [Internet]. 2006 Jan [cited 2012 Jul 15];45(1):66-71. Available from: http:// www.ncbi.nlm.nih.gov/pubmed/16263782.

120. El Miedany Yasser, El Gaafary Maha, Youssef S AI. Validity of the developed Arabic Multidimensional Health Assessment Questionnaire for use in standard clinical care of patients with rheumatic diseases. International Journal of Rheumatic Diseases. 2008;11(3):224-36.

121. Prajapati R. Investigation of the relationship between patient-centered factors and baseline disease activity (DAS28) in rheumatoid arthritis (RA). Arthritis and Rheumatism. 2011;63(10).

122. Koh E. T, Seow A, Pong LY, Koh WH, Chan L, Howe $\mathrm{HS}$, et al. Cross cultural adaptation and validation of the Chinese Health Assessment Questionnaire for use in rheumatoid arthritis. The Journal of rheumatology [Internet]. 1998 Sep [cited 2012 Nov 6];25(9):1705-8. Available from: http://www.ncbi.nlm.nih.gov/pubmed/9733449.

123. Archenholtz B, Dellhag B. Validity and reliability of the instrument Performance and Satisfaction in Activities of Daily Living (PS-ADL) and its clinical applicability to adults with rheumatoid arthritis. Scandinavian journal of occupational therapy [Internet]. 2008 Mar [cited 2012 Jan 20];15(1):13-22. Available from: http://www.ncbi. nlm.nih.gov/pubmed/17852967.

124. Riazzoli J, Nilsson J-Å, Teleman A, Petersson IF, RantapääDahlqvist S, Jacobsson LTH, et al. Patient-reported 28 swollen and tender joint counts accurately represent RA disease activity and can be used to assess therapy responses at the group level. Rheumatology (Oxford, England) [Internet]. 2010 Nov [cited 2012 Nov 5];49(11):2098-103. 
Available from: http://www.ncbi.nlm.nih.gov/pub$\mathrm{med} / 20615918$.

125. Munters LA. Patient Preference of Disability in Rheumatoid Arthritis. [abstract]. Arthritis Rheum. 2011;63(suppl 10):2424.

126. Fransen J, Langenegger T, Michel B a, Stucki G. Feasibility and validity of the RADAI, a self-administered rheumatoid arthritis disease activity index. Rheumatology (Oxford, England) [Internet]. $2000 \mathrm{Mar} ; 39(3): 321-7$. Available from: http://www.ncbi.nlm.nih.gov/pubmed/10788543.

127. Fransen J, Häuselmann H, Michel BA, Caravatti M, Stucki G. Responsiveness of the self-assessed rheumatoid arthritis disease activity index to a flare of disease activity. Arthritis and rheumatism [Internet]. 2001 Jan [cited 2012 Apr 23];44(1):53-60. Available from: http://www.ncbi.nlm. nih.gov/pubmed/11212176.

128. Durmus B, Altay Z, Baysal O, Ersoy Y, Erdal A, Cevik $\mathrm{R}$, et al. Can the patient-reported outcome instruments determine disease activity in rheumatoid arthritis? Bratislavské lekárske listy [Internet]. 2011 Jan [cited 2012 Nov 2];112(10):555-61. Available from: http://www.ncbi.nlm. nih.gov/pubmed/21954539.

129. Köybaşi M. Problems of self-care activities encountered in rheumatoid arthritis and their relationship with disease activity and hand. Turkish Journal of Rheumatology. 2011;26(2).

130. Amirdzhanova VN, Folomeeva OM, Tsvetkova ES, Aleksandrov AA. [Use of modified Stanford Health Assessment Questionnaire in patients with rheumatoid arthritis]. Revmatologiia (Moscow, Russia) [Internet]. [cited 2012 Nov 5];(3):56-61. Available from: http:/www.ncbi.nlm. nih.gov/pubmed/2609061.

131. Szilasiová A, Macejová Z, Nagyová I, Kovarová M, Béresová A, Szilasiová J. [Reliability and validation of the Slovak modified version of the Stanford Health Assessment Questionnaire using the functional disability index in patients with rheumatoid arthritis]. Vnitr囚ní lékar\ství [Internet]. 2002 Jan [cited 2012 Nov 5];48(1):8-16. Available from: http://www.ncbi.nlm.nih.gov/pubmed/11852593.

132. Goeppinger J, Doyle MA, Charlton SL, Lorig K. A nursing perspective on the assessment of function in persons with arthritis. Research in nursing \& health [Internet]. 1988 Oct [cited 2012 Nov 5];11(5):321-31. Available from: http://www.ncbi.nlm.nih.gov/pubmed/3175056.

133. Clarke AE, Fries JF. Health status instruments and physical examination techniques in clinical measurement methodologies. Current opinion in rheumatology [Internet]. 1992 Apr [cited 2012 Nov 5];4(2):145-52. Available from: http://www.ncbi.nlm.nih.gov/pubmed/1581141.

134. Pincus T, Segurado OG. Most visits of most patients with rheumatoid arthritis to most rheumatologists do not include a formal quantitative joint count. Annals of the rheumatic diseases [Internet]. 2006 Jun [cited 2012 Jan 20];65(6):820-2. Available from: http://www.pubmedcentral.nih.gov/articlerender.fcgi? artid=1798189\& 2 tool $=$ pmcentrez\&rendertype $=$ abstract.
135. Pincus T, Oliver AM, Bergman MJ. How to collect an MDHAQ to provide rheumatology vital signs (function, pain, global status, and RAPID3 scores) in the infrastructure of rheumatology care, including some misconceptions regarding the MDHAQ. Rheumatic diseases clinics of North America [Internet]. 2009 Nov [cited 2012 Jan 20];35(4):799-812, x. Available from: http://www.ncbi. nlm.nih.gov/pubmed/19962624.

136. Patrick DL, Burke LB, Powers JH, Scott JA, Rock EP, Dawisha $S$, et al. Patient-reported outcomes to support medical product labeling claims: FDA perspective. Value in health: the journal of the International Society for Pharmacoeconomics and Outcomes Research [Internet]. [cited 2012 Jul 22];10 Suppl 2:S125-37. Available from: http://www.ncbi.nlm.nih.gov/pubmed/17995471.

137. Fiscella K, Ransom S, Jean-Pierre P, Cella D, Stein K, Bauer JE, et al. Patient-reported outcome measures suitable to assessment of patient navigation. Cancer [Internet]. 2011 Aug [cited 2012 Jul 22];117(15 Suppl):3603-17. Available from: http://www.ncbi.nlm.nih.gov/pubmed/21780095.

138. Yost KJ, Eton DT, Garcia SF, Cella D. Minimally important differences were estimated for six Patient-Reported Outcomes Measurement Information System-Cancer scales in advanced-stage cancer patients. Journal of clinical epidemiology [Internet]. 2011 May [cited 2012 Jul 22];64(5):507-16. Available from: http://www.pubmedcentral.nih.gov/articlerender.fcgi?artid $=3076200 \&$ tool $=p$ mcentrez\&rendertype $=$ abstract.

139. Rothrock NE, Hays RD, Spritzer K, Yount SE, Riley W, Cella D. Relative to the general US population, chronic diseases are associated with poorer health-related quality of life as measured by the Patient-Reported Outcomes Measurement Information System (PROMIS). Journal of clinical epidemiology [Internet]. 2010 Nov [cited 2012 Jul 22];63(11):1195-204. Available from: http://www. pubmedcentral.nih.gov/articlerender.fcgi? artid $=2943571$ \&tool=pmcentrez\&rendertype $=$ abstract.

140. Yeatts KB, Stucky B, Thissen D, Irwin D, Varni JW, DeWitt EM, et al. Construction of the Pediatric Asthma Impact Scale (PAIS) for the Patient-Reported Outcomes Measurement Information System (PROMIS). The Journal of asthma : official journal of the Association for the Care of Asthma [Internet]. 2010 Apr [cited 2012 Jul 22];47(3):295-302. Available from: http://www.pubmedcentral.nih.gov/articlerender.fcgi?artid=3098738\&tool= pmcentrez\&rendertype $=$ abstract .

141. Pincus T, Yazici Y, Bergman M. A practical guide to scoring a Multi-Dimensional Health Assessment Questionnaire (MDHAQ) and Routine Assessment of Patient Index Data (RAPID) scores in 10-20 seconds for use in standard clinical care, without rulers, calculators, websites or computers. Best practice \& research. Clinical rheumatology [Internet]. 2007 Aug [cited 2012 Jan 20];21(4):755-87. Available from: http://www.ncbi.nlm.nih.gov/pubmed/17678834.

142. Goodacre L, Smith J, Meddis D, Goodacre J. Development and validation of a patient-centred Measure of Activity 
Limitation (MAL) in rheumatoid arthritis. Rheumatology (Oxford, England) [Internet]. 2007 Apr [cited 2012 Jun 26];46(4):703-8. Available from: http://www.ncbi.nlm. nih.gov/pubmed/17166868.

143. Pincus T, Sokka T, Kautiainen H. Further development of a physical function scale on a MDHAQ [corrected] for standard care of patients with rheumatic diseases. The Journal of rheumatology [Internet]. 2005 Aug [cited 2012 Apr 24];32(8):1432-9. Available from: http://www.ncbi. nlm.nih.gov/pubmed/16078316.

144. Pincus T, Sokka T. Laboratory tests to assess patients with rheumatoid arthritis: advantages and limitations. Rheumatic diseases clinics of North America [Internet]. $2009 \mathrm{Nov}$ [cited 2012 Jan 20];35(4):731-4, vi-vii. Available from: http://www.ncbi.nlm.nih.gov/pubmed/19962617.

145. Pincus T, Swearingen C, Wolfe F. Toward a multidimensional Health Assessment Questionnaire (MDHAQ): assessment of advanced activities of daily living and psychological status in the patient-friendly health assessment questionnaire format. Arthritis and rheumatism [Internet]. 1999 Oct [cited 2012 Feb 21];42(10):2220-30. Available from: http://www.ncbi.nlm.nih.gov/pubmed/10524697.

146. Pincus T. Advantages and limitations of quantitative measures to assess rheumatoid arthritis: joint counts, radiographs, laboratory tests, and patient questionnaires. Bulletin of the NYU hospital for joint diseases [Internet]. 2006 Jan [cited 2012 Apr 25];64(1-2):32-9. Available from: http://www.ncbi.nlm.nih.gov/pubmed/17121487.

147. Lubeck DP. Patient-reported outcomes and their role in the assessment of rheumatoid arthritis. PharmacoEconomics [Internet]. 2004 Jan [cited 2012 Apr 23];22(2 Suppl 1):27-38. Available from: http://www.ncbi.nlm.nih.gov/ pubmed/15157002.

148. Gossec L. Patient-Reported Outcomes in Rheumatoid Arthritis: Why are They Important and How Should They Be Assessed? Turkish Journal of Rheumatology [Internet]. 2010 Sep 1 [cited 2012 Jan 20];25(3):99-104. Available from: http://tjr.org.tr/text.php3?id=355.

149. Garip Y, Eser F, Bodur H. Health-related quality of life in rheumatoid arthritis: comparison of RAQoL with other scales in terms of disease activity, severity of pain, and functional status. Rheumatology international [Internet]. 2011 Jun [cited 2012 Apr 22];31(6):769-72. Available from: http://www.ncbi.nlm.nih.gov/pubmed/20180124.

150. Pincus T, Brooks RH, Callahan LF. Prediction of long-term mortality in patients with rheumatoid arthritis according to simple questionnaire and joint count measures. Annals of internal medicine [Internet]. 1994 Jan 1;120(1):26-34. Available from: http://www.ncbi.nlm.nih.gov/pubmed/8250453.

151. Callahan LF, Pincus T, Huston JW, Brooks RH, Nance EP, Kaye JJ. Measures of activity and damage in rheumatoid arthritis: depiction of changes and prediction of mortality over five years. Arthritis care and research [Internet]. 1997 Dec [cited 2012 Jul 23];10(6):381-94. Available from: http://www.ncbi.nlm.nih.gov/pubmed/9481230.
152. Sokka T, Häkkinen a, Krishnan E, Hannonen P. Similar prediction of mortality by the health assessment questionnaire in patients with rheumatoid arthritis and the general population. Annals of the rheumatic diseases [Internet]. 2004 May [cited 2011 Dec 18];63(5):494-7. Available from: http://www.pubmedcentral.nih.gov/articlerender.fc gi?artid $=1754988 \&$ tool $=$ pmcentrez\&rendertype $=$ abstract.

153. Wolfe F, Michaud K, Gefeller O, Choi HK. Predicting mortality in patients with rheumatoid arthritis. Arthritis and rheumatism [Internet]. 2003 Jun [cited 2012 Jul 23];48(6):1530-42. Available from: http://www.ncbi.nlm. nih.gov/pubmed/12794820.

154. Pincus T, Yazici Y, Bergman M, Maclean R, Harrington T. A proposed continuous quality improvement approach to assessment and management of patients with rheumatoid arthritis without formal joint counts, based on quantitative routine assessment of patient index data (RAPID) scores on a multidimensional health a. Best practice $\&$ research. Clinical rheumatology [Internet]. 2007 Aug [cited 2012 Jan 20];21(4):789-804. Available from: http://www.ncbi. nlm.nih.gov/pubmed/17678835.

155. Blanchais A, Berthelot J-M, Fontenoy A-M, Le Goff B, Maugars Y. Weekly home self-assessment of RAPID-4/3 scores in rheumatoid arthritis: a 6-month study in 26 patients. Joint, bone, spine : revue du rheumatism [Internet]. Elsevier Masson SAS; 2010 Dec [cited 2012 Jan 20];77(6):582-7. Available from: http://www.ncbi.nlm. nih.gov/pubmed/20889362.

156. Yazici Y, Bergman M, Pincus T. Time to score quantitative rheumatoid arthritis measures: 28-Joint Count, Disease Activity Score, Health Assessment Questionnaire (HAQ), Multidimensional HAQ (MDHAQ), and Routine Assessment of Patient Index Data (RAPID) scores. The Journal of rheumatology [Internet]. 2008 Apr [cited 2012 Apr 24];35(4):603-9. Available from: http://www. ncbi.nlm.nih.gov/pubmed/18322993.

157. Wolfe F, Pincus T, Fries JF. Usefulness of the HAQ in the clinic. Annals of the rheumatic diseases [Internet]. 2001 Aug [cited 2012 Apr 24];60(8):811. Available from: http:// www.pubmedcentral.nih.gov/articlerender.fcgi?artid=175 3803\&tool=pmcentrez\&rendertype $=$ abstract.

158. Fries JF, Spitz P, Kraines RG, Holman HR. Measurement of patient outcome in arthritis. Arthritis and rheumatism [Internet]. 1980 Feb [cited 2012 Jan 11];23(2):137-45. Available from: http://www.ncbi.nlm.nih.gov/pub$\mathrm{med} / 7362664$.

159. Pincus T, Askanase AD, Swearingen CJ. A multi-dimensional health assessment questionnaire (MDHAQ) and routine assessment of patient index data (RAPID3) scores are informative in patients with all rheumatic diseases. Rheumatic diseases clinics of North America [Internet]. 2009 Nov [cited 2012 Jan 20];35(4):819-27, x. Available from: http://www.ncbi.nlm.nih.gov/pub$\mathrm{med} / 19962626$.

160. Hogrefe JF, Marengo MF, Schneerberger EE, Rosemffet M, Cocco JCM, Citera G. Valor de corte de HAQ para 
predecir discapacidad laboral en pacientes con artritis reumatoidea. Rev Arg Reumatol. 20(2):23-7.

161. Pincus T, Yazici Y, Sokka T. Quantitative measures of rheumatic diseases for clinical research versus standard clinical care: differences, advantages and limitations. Best practice \& research. Clinical rheumatology [Internet]. 2007 Aug [cited 2012 Jan 20];21(4):601-28. Available from: http:// www.ncbi.nlm.nih.gov/pubmed/17678823.

162. Aletaha D, Landewe R, Karonitsch T, Bathon J, Boers $\mathrm{M}$, Bombardier C, et al. Reporting disease activity in clinical trials of patients with rheumatoid arthritis: EULAR/ACR collaborative recommendations. Arthritis and rheumatism [Internet]. 2008 Oct 15 [cited 2011 Dec 28];59(10):1371-7. Available from: http://www.ncbi.nlm. nih.gov/pubmed/18821648.

163. Pincus T, Strand V, Koch G, Amara I, Crawford B, Wolfe F, et al. An index of the three core data set patient questionnaire measures distinguishes efficacy of active treatment from that of placebo as effectively as the American College of Rheumatology 20\% response criteria (ACR20) or the Disease Activity Score (DAS) in Arthritis and rheumatism [Internet]. 2003 Mar [cited 2012 Jan 20];48(3):625-30. Available from: http://www.ncbi.nlm. nih.gov/pubmed/12632413.

164. Pincus T, Chung C, Segurado OG, Amara I, Koch GG. An index of patient reported outcomes (PRO-Index) discriminates effectively between active and control treatment in 4 clinical trials of adalimumab in rheumatoid arthritis. The Journal of rheumatology [Internet]. $2006 \mathrm{Nov}$ [cited 2012 Apr 25];33(11):2146-52. Available from: http:// www.ncbi.nlm.nih.gov/pubmed/17080518.

165. Pincus T, Yazici Y, Bergman MJ. Beyond RAPID3 - practical use of the MDHAQ to improve doctor-patient com- munication. Bulletin of the NYU hospital for joint diseases [Internet]. 2010 Jan [cited 2012 Apr 24];68(3):223-31. Available from: http://www.ncbi.nlm.nih.gov/pub$\mathrm{med} / 20969556$.

166. Pincus T, Keysor J, Sokka T, Krishnan E, Callahan LF. Patient questionnaires and formal education level as prospective predictors of mortality over 10 years in $97 \%$ of 1416 patients with rheumatoid arthritis from 15 United States private practices. The Journal of rheumatology [Internet]. 2004 Feb [cited 2012 Apr 22];31(2):229-34. Available from: http://www.ncbi.nlm.nih.gov/pubmed/14760789.

167. Sokka T. Assessment of pain in rheumatic diseases. Clinical and experimental rheumatology [Internet]. 2005 [cited 2012 Mar 5];23(5 Suppl 39):S77-84. Available from: http://www.ncbi.nlm.nih.gov/pubmed/16273789.

168. Studenic P, Radner H, Smolen JS, Aletaha D. Discrepancies between patients and physicians in the perception of rheumatoid arthritis disease activity. Arthritis and rheumatism [Internet]. 2012 Jul 18 [cited 2012 Jul 25]; Available from: http://www.ncbi.nlm.nih.gov/pubmed/22810704.

169. Pincus T, Sokka T. Quantitative measures for assessing rheumatoid arthritis in clinical trials and clinical care. Best practice $\&$ research. Clinical rheumatology [Internet]. 2003 Oct [cited 2012 Apr 25];17(5):753-81. Available from: http://linkinghub.elsevier.com/retrieve/ pii/S1521694203000779.

170. Cardiel MH, Abello-Banfi M, Ruiz-Mercado R, AlarconSegovia D. How to measure health status in rheumatoid arthritis in non-English speaking patients: validation of a Spanish version of the Health Assessment Questionnaire Disability Index (Spanish HAQ-DI). Clinical and experimental rheumatology. 11(2):117-21. 